\title{
Interpret The Synergistic Mechanism of Shenfu Decoction in Treating Sick Sinus Syndrome From the Perspective of Systematic Pharmacology
}

\section{Yihua Li ( $\square$ gzylyh01@163.com )}

Guangzhou University of Chinese Medicine https://orcid.org/0000-0002-9056-2386

\section{Zhiyun Luo}

Guangzhou University of Chinese Medicine

\section{Yupeng Chen}

Southern Medical University

\section{Kexin Wang}

Shanxi University, Taiyuan City

Yi Li

Southern Medical University

Jieqi Cai

Southern Medical University

\section{Xinjun Zhao}

Guangzhou University of Traditional Chinese Medicine First Affiliated Hospital

\section{Qingmin Chu}

Guangzhou University of Traditional Chinese Medicine First Affiliated Hospital

\section{Daogang Guan}

Southern Medical University

\section{Rong Li}

Guangzhou University of Traditional Chinese Medicine First Affiliated Hospital

\section{Research}

Keywords: Shenfu Decoction, Sick Sinus Syndrome, systematic pharmacology, molecular docking

Posted Date: December 23rd, 2020

DOI: https://doi.org/10.21203/rs.3.rs-132215/v1

License: (1) (1) This work is licensed under a Creative Commons Attribution 4.0 International License.

Read Full License 


\title{
Interpret the Synergistic Mechanism of Shenfu Decoction in Treating Sick Sinus Syndrome from the Perspective of Systematic Pharmacology
}

Yihua Li ${ }^{\mathrm{a}}$, Zhiyun Luo ${ }^{\mathrm{a}}$, Yupeng Chen ${ }^{\mathrm{b}, \mathrm{c}}$, Kexin Wangd, e, Yi Lif, Jieqi Cai ${ }^{\mathrm{b}, \mathrm{c}}$, Xinjun Zhao, Qingmin Chu ${ }^{g}$, Daogang Guan ${ }^{b, c^{*}}$, Rong Li ${ }^{*}$

${ }^{a}$ Guangzhou University of Chinese Medicine, Guangzhou, 510000, China

${ }^{b}$ Department of Biochemistry and Molecular Biology, School of Basic Medical Sciences, Southern Medical University, Guangzhou, 510000, China

'Guangdong Key Laboratory of Single Cell Technology and Application, Southern Medical University, Guangzhou, 510000, China

dModern Research Center for Traditional Chinese Medicine, Shanxi University, Taiyuan, 030000, China

eInstitute of Integrated Bioinformedicine and Translational Science, Hong Kong Baptist University, Hong Kong, 999077, Hong Kong

fDepartment of Radiology, Nanfang Hospital, Southern Medical University, Guangzhou, 510000, China

gDepartment of Cardiovascular Disease, First Affiliated Hospital of Guangzhou University of Chinese Medicine, Guangzhou, 510000, China

* Corresponding authors. E-mail addresses: guanyufei122@163.com (D. Guan), 1rhbs@126.com (R. Li).

\begin{abstract}
Introduction: Sick sinus syndrome (SSS) is a common cardiovascular disease without available oral drugs for a long-term routine treatment, which can be treated by Shenfu Decoction (SFD) according to Traditional Chinese Medicine (TCM) clinical applications. The purpose of this research is to decode the therapeutic mechanisms of SFD in treating SSS as well as interpret the synergistic relationship of herbs in SFD through the method of systematic pharmacology.

Methods: We established a novel systematic pharmacological model, which integrate pharmacokinetic parameters, pharmacological data and network topology analysis, to effectively screen the key components of SFD. Based on the key components, gene ontology, pathway enrichment and molecular docking were used to clarify and validate the therapeutic mechanisms of
\end{abstract}


SFD in treating SSS and the synergistic relationship of herbs in SFD.

Results: Through our proposed mathematical model, we retrieved 55 components which were defined as synergistic functional components group (SFCG) from 209 potential active components of SFD screened by ADME parameters. Pathway analysis of SFCG targets indicated that RS and FZ could synergistically target to neuroactive ligand-receptor reaction, cAMP signaling pathway, calcium signaling pathway, PI3K-Akt signaling pathway, Rap1 signaling pathway and other pathways to treat SSS. Additionally, molecular docking analysis showed that the average affinity of SFCG and targets involved in the key pathways reached $-6.36 \mathrm{kcal} / \mathrm{mmol}$, validating the synergistic effect of RS and FZ in treating SSS.

Conclusions: The therapeutic effect of SFD on SSS may be due to its functions of regulating ion channel function, signal transduction and energy metabolism. Besides, we found that the synergistic relationship between RS and FZ can be described at the levels of target, function and pathway.

Keywords: Shenfu Decoction, Sick Sinus Syndrome, systematic pharmacology, molecular docking

\section{Introduction}

Sick sinus syndrome (SSS) is a clinical syndrome caused by structural changes and dysfunction of sinus node, which can lead to a variety of arrhythmias, such as sinus bradycardia, sinoatrial block, sinus arrest, tachycardia-bradycardia syndrome, and etc. Its clinical manifestations mainly include palpitations, dizziness, syncope, and in serious cases, it can lead to Adams-Stokes syndrome or sudden death [1]. Degenerative fibrosis of the sinoatrial node, ion channel dysfunction and remodeling of the sinoatrial node are considered as the most important pathogenesis of SSS [2]. In addition, SSS is generally a disease of aging with a slow process of occurrence and development. With the increase of age, the sinus node presents non-specific degenerative fibrosis, which is an important factor leading to SSS. Besides that, diseases such as coronary heart disease, cardiomyopathy and myocarditis are also common causes of SSS [2] and may lead to the reduction of normal sinoatrial node cells, which is one of the most important pathological features of SSS [1].

For the treatment of SSS, commonly used drugs include atropine and isoproterenol, but they can only be used for symptomatic treatment. These drugs are not suitable enough for long-term used due to their short duration of efficacy and numerous adverse reactions [3]. Permanent pacemaker placement is recommended in patients with clinical indications, but the invasive and expensive features of it limits the clinical applications. Therefore, seeking a safe, effective and suitable drug for long-term used has become an urgent and difficult problem to be solved. Excitingly, Traditional Chinese Medicine (TCM) with a history of thousands of years provides valuable ideas for SSS treatment. According to the theories of TCM, SSS is mainly attributed to deficiency of heart yang, in other words, decline of the normal pulsatile function of the heart. In clinical practice, yang warming herbs and prescriptions, such as Shenfu Decoction (SFD) and Mahuang Fuzi Xixin Decoction, are often used to treat SSS, for they are considered that can stimulate or restore the 
normal physiological function of the heart.

SFD is a famous TCM prescription, which has been used to treat heart diseases for about hundreds of years in China. It is composed of Panax ginseng C.A.Mey (RS) and Radix Aconiti Lateralis Preparata (FZ) with a dosage ratio of 1:2. According to the TCM theory, RS has the function of powerfully supplementing original $q i$, and $\mathrm{FZ}$ can supplement fire and assist yang, they can achieve a synergistic effect in this prescription. When they are used together, they can play the role of boosting $q i$ and warming yang to restore cardiac function, which is very suitable for SSS patients caused by yang deficiency. Increasing modern medical researches show that SFD-based treatment of SSS can not only improve sinus node function and heart rate, but also increase coronary artery blood flow to improve sinus node blood supply [4,5,6]. Additionally, modern pharmacological studies have found that the main active components of SFD are Ginsenosides and Aconitines. Ginsenosides are reported to excite myocardium, increase myocardial contractility, promote cardiac output, improve cardiac function and dilate coronary artery, etc. Aconitine can improve cardiac electrophysiological conduction block and accelerate heart rate, thus achieving the effect of anti-bradycardia [7]. In addition, Shenfu Injection (SFI) prepared by SFD extraction (product officially approved in 1987, approval code: certification number Z 20043117; Standard: $50 \mathrm{ml}$ per bottle [8] is also widely used for SSS treatment in China. Moreover, published reports have shown that SFI can not only effectively improve the heart rate of patients, but also promote the recovery of sinus node function [9], and the clinical efficacy is even better than atropine [10]. It can be seen from the above that the therapeutic effect of SFD in treating SSS is confirmed. However, due to the complex chemical compositions of SFD, the specific molecular mechanism of its treatment on SSS is difficult to be clarified. Therefore, we introduced the method of network pharmacology to capture the potential synergistic mechanism.

Network pharmacology is an important product in the rapid development of bioinformatics, and is a useful tool for describing the mechanism of complex drug systems in detail [11]. It can clarify the complex relationship between herbal ingredients and diseases in a computable form at the molecular level through the network of "ingredients-targets-diseases" [12]. Compared with the traditional pharmacology model of "one drug, one target", network pharmacology pays more attention to integrity and systematicness between drugs and disease, and emphasizes the network relationship of "multiple components and multiple targets", which coincides with the core concept of TCM. Through network pharmacology research, we can predict the targets and pharmacological effects of herbal compounds to reveal the overall effects of herbal prescriptions, thus providing ideas for further researches.

Here, we designed a network pharmacology method, which combined a synergistic influential contribution (SIC) model and a target coverage optimization (TCO) model to explore the synergistic molecular mechanisms of SFD in treating SSS, which could lay a theoretical basis for clinical application of SFD and provide an idea for prescription rules explanation of TCM formulas.

\section{Methods}




\subsection{Chemical component database}

The chemical components of RS and FZ were obtained from TCMSP database ( http://tcmspw.com/tcmsp.php ） , ETCM database (http://www.nrc.ac.cn:9090/ETCM/index.php/Home/Index/index.html) and TCMID database (http://119.3.41.228:8000/tcmid/) respectively. After integration and elimination of duplication, the canonical SMILES of each component was obtained from Pubchem database (https://pubchem.ncbi.nlm.nih.gov/) .

\subsection{ADME screening}

The ADME parameters of each component, including Druglikeness, GI Absorption and Bioavailability Score, were calculated in SwissADME database （http://www.swissadme.ch/） by using the canonical SMILES.

Druglikeness refers to the similarity between components and known drugs. Drug-like components are not drugs, but have the possibility of becoming drugs. Such components are called drug-like molecules or drug analog molecules. In drug developments, druglikeness means component with suitable drug properties for lead compound researches, so it can be said that druglike compounds are high-quality lead compounds. In this study, Lipinski principle (MW $\leq 500$, MLOGP $\leq 4.15, \mathrm{~N}$ or $\mathrm{O} \leq 10, \mathrm{NH}$ or $\mathrm{OH} \leq 5$ ) was used to analyze the drug-like properties of each component, and components meeting this principle will be retained for further analysis.

Gastrointestinal Absorption (GI Absorption) is a kind of pharmacokinetic behavior, which is one of the key factors that determine the bioavailability of drugs. It is a very important parameter of drugs during different stages of drug discovery. A novel BOILED-Egg model, distinguishing the absorption of molecules based on their lipophilicity and polarity, is used to evaluate the GI Absorption of each molecule on the SwissADME database [13]. And for all components of RS and FZ, those with 'High' GI Absorption will be retained.

Bioavailability refers to the speed and degree of drug absorption into blood circulation, which reflects the percentage of oral drug dosage absorbed by gastrointestinal tract and reaching blood circulation after passing through liver [14]. In this study, components with Bioavailability scores greater than or equal to 0.55 will be retained.

\subsection{Target prediction}

The public databases SwissTargetPrediction(http://www.swisstargetprediction.ch/), Similarity Ensemble Approach ( http://sea.bkslab.org/ ) and HitPick ( http://mips.helmholtzmuenchen.de/hitpick/cgi-bin/index.cgi?content=targetPrediction.html) are used to predict the target of each component. In addition, the GeneCards database （https://www.genecards.org/） is used to obtain the relevant targets of SSS and their relevant scores.

\subsection{Gene Ontology and pathway analysis}

In order to explore the potential functions of SFD in treating SSS, we use clusterProfiler, an R 
language package [15], to carry out the Gene Ontology (GO) and KEGG pathway enrichment analysis. Results of GO or KEGG analysis with the P-value less than or equal to 0.05 are retained for the further analysis.

\subsection{SIC and TCO calculation}

The SIC and TCO are important parameters of the active components, which are used to assess the importance or contribution of an active component in the C-T network as well as its effectiveness in the treatment of SSS. In order to evaluate the therapeutic effect of SFD on SSS, we established a mathematical formula to calculate the SIC and TCO values of each active component in SFD to find the key components which are defined as synergistic functional components group (SFCG):

$$
\begin{gathered}
\theta_{i}=\frac{B}{S} \\
C_{i j}=\theta_{i}+\left|\frac{B_{R i}+B_{F i}}{B_{R i}-B_{F i}}\right| \\
\operatorname{SIC}(i)=\sum_{i j}^{n}\left(B_{i} \times\left[C_{i j} \times D_{j}\right]\right) \\
\operatorname{TCO}(i)=\frac{X i}{\sum_{i=1}^{n}(X i)} \\
\operatorname{SFCG}=90 \% S I C \cap 90 \% T C O
\end{gathered}
$$

B stands for the Betweenness Centrality of each active component, and S stands for the sum of the Betweenness Centrality of the targets corresponding to each active component. $B_{R}$ refers to the Betweenness Centrality of active components in RS and $\mathrm{B}_{\mathrm{F}}$ represents the Betweenness Centrality of active components in FZ. $\left|\frac{B_{R i}+B_{F i}}{B_{R i}-B_{F i}}\right|$ is 0 if an active component exists only in one herb. $\mathrm{C}_{i j}$ is the affinity index determined according to $\theta_{i}$. i represents the number of SFD active components, and $j$ represents the predicted target number of active components. The Betweenness Centrality of active components and targets was calculated by Cytoscape 3.7.2 [16]. D represents the degree of each target. X represents the count of targets corresponded to an active component. 90\% TCO represents targets with a cumulative coverage rate of $90 \%$, while $90 \%$ SIC represents targets with a cumulative synergistic rate of $90 \%$.

2.6. Molecular docking validation analysis

Molecular docking was used to evaluate the interactions between components of SFCG and key targets involved in major pathways. Firstly, the 3D structure files of the SFCG were retrieved and acquired by PubChem, ZINC (http://zinc.docking.org/) and TCMSP databases, and then were transformed into *.pdb format files by using OpenBabel software [17], thus constituting the ligands 
library. Meanwhile, the 3D structure files of key targets were obtained through PDB (https://www.rcsb.org/) database and constructed the receptors library. Then, Raccoon software [18] was used for batch pretreatment of ligands and receptors, including adding Gasteiger charges, cleaning up non-polar hydrogen and deleting lone pairs for ligands and removing non-polar hydrogen and water molecules for receptors. Then, Autodock Vina [19] was used for semi-flexible docking of ligands and receptors, and the docking results were integrated and analyzed by PLIP [20] (https://projects.biotec.tu-dresden.de/plip-web/plip). At last, PyMOL and LigPlot+ [21] were used to visually analyze the interactions of ligands and receptors.

\section{Results}

In this study, the synergistic mechanisms of SFD in treating SSS are clarified by using systematic pharmacological model. Firstly, all components of SFD are collected from TCMSP database, ETCM database and TCMID database. Secondly, the potential active components are screened by ADME rules. Thirdly, three online tools are used to predict the targets of each active component. Then the components and their targets are used to construct C-T network, based on which the SIC and TCO of all components were calculated to estimate the influence of SFCG in the treatment of SSS. Finally, the SFCG and their targets were used to decode and validate the synergistic molecular mechanisms of SFD in treating SSS (Fig. 1).

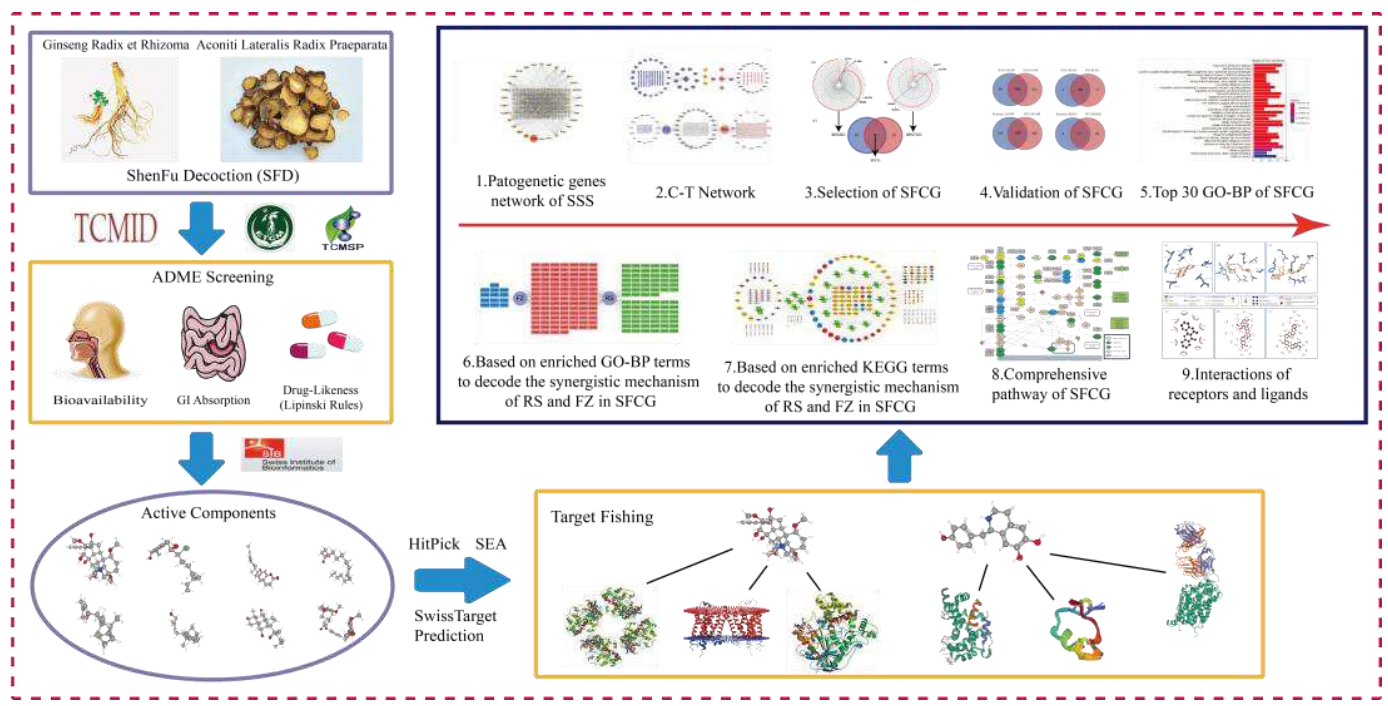

Fig. 1. The flow chart of systematic pharmacology to decode the synergistic mechanism of SFD in treating SSS.

\subsection{Pathogenetic genes analysis of SSS}

It had been known that the etiology of SSS mainly includes aging, coronary heart disease, cardiomyopathy, etc. Natural aging of human leads to fibrosis or programmed apoptosis of sinoatrial node cells, coronary heart disease leads to sinoatrial node ischemia, both of them cause gradual 
decrease of sinoatrial node cells and dysfunction of ion channels in the heart, which are currently recognized as the main pathogenesis mechanisms of SSS. In this study, a total of 900 SSS-related pathogenic genes and their relevant scores were obtained from GeneCards database, as shown in Table S1. Then, we use STRING database to analyze the gene interaction of SSS pathogenic genes, and the results are shown in Fig. 2.

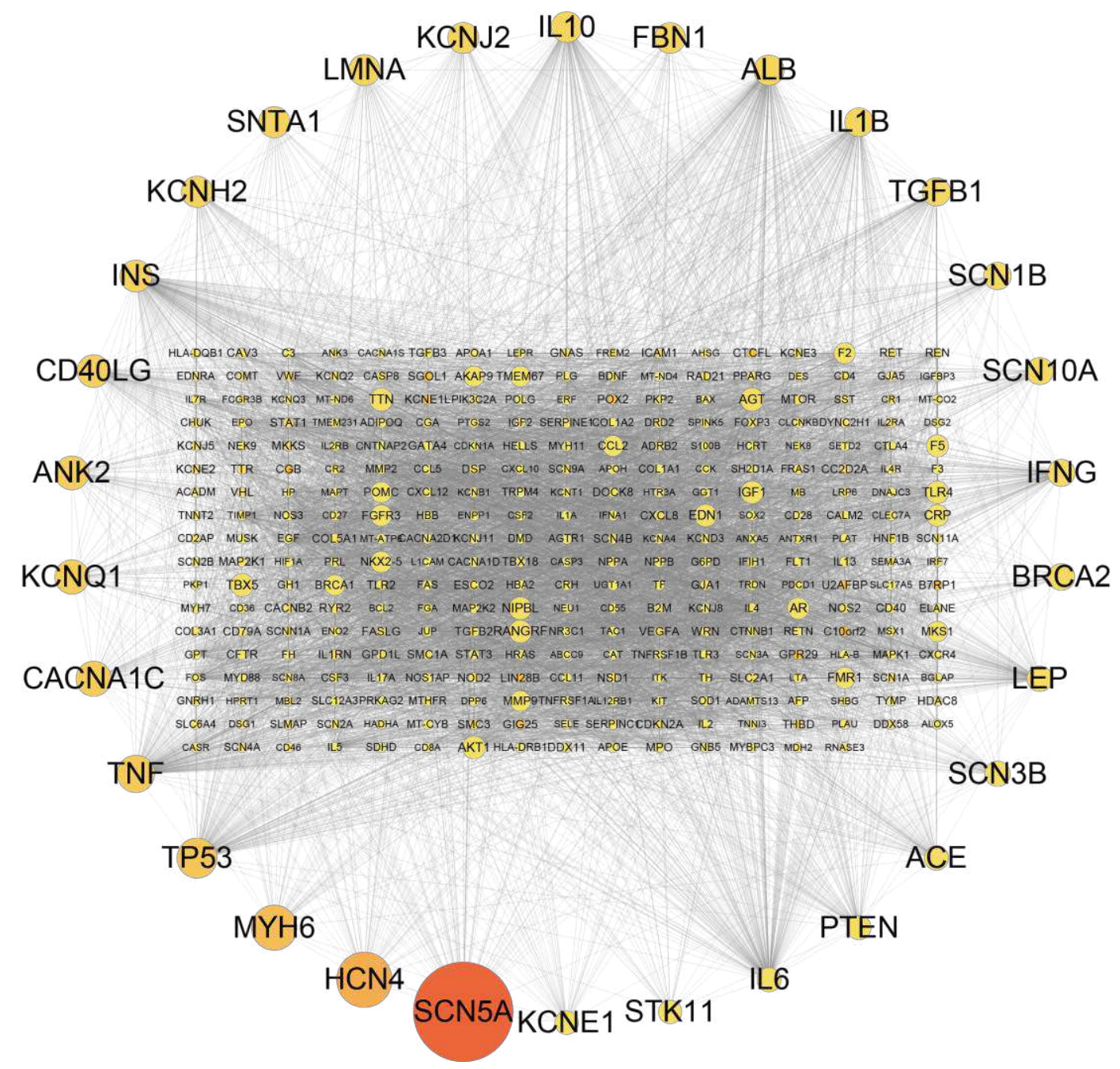

Fig. 2. The weighted pathogenesis genes network of SSS. The node size means the weight of the node, which quantified by relevant score.

Among these genes, SCN5A, HCN4, MYH6, TP53, TNF and CACNA1C have highest relevant score, it worth to note that most of these genes are reported to be associated with pathogenesis or therapy of SSS. SCN5A encodes Sodium Voltage-Gated Channel Alpha Subunit 5 , mutations of which will decrease the inward $\mathrm{Na}^{+}$current and result in slowing down pacemaking and cardiac conduction [22]. HCN4 is an important subtype of $\mathrm{HCN}$ family and is mainly expressed in sinoatrial node. It encodes cardiac pacing current $\mathrm{I}_{\mathrm{f}}$ and plays an important role in regulating heart rate 23]. MYH6 encodes Myosin Heavy Chain 6, which is significant for myocardial contraction. It is reported that a rare missense of MYH6 associates with high risk of 
SSS [24]. TP53 protein ubiquitously expresses in non-myocyte cells in the heart and plays an important role in the regulation of cardiomyocyte apoptosis [25]. TNF encodes a multifunctional proinflammatory cytokine that belongs to the tumor necrosis factor superfamily and it can activate negative inotropic effects and apoptosis in myocytes [26]. CACNA1C encodes Calcium VoltageGated Channel Subunit Alpha1 C and mediates the influx of calcium ions into the cell upon membrane polarization, reduced expression of which can lead to sinus node dysfunction and slow down pacemaking [27]. These results indicate that pathogenetic genes we selected are more reliable for characterizing the pathogenesis or therapy targets of SSS.

\subsection{Potential active components in RS and FZ}

Similar to other TCM preparations, SFD has a series of components, while only a few of them have satisfactory pharmacodynamic and pharmacokinetic characteristics. In this study, 297 components of RS and 96 of FZ were obtained through database retrieval, refers to Table S2. Then, three parameters were used for ADME screening, but during ADME screening, we found that all the Ginsenosides were screened out, while many pharmacological studies showed that Ginsenosides were the main active components of RS [28], so all the Ginsenosides we retrieved were also added to the list of active components of RS. Thus 209 active components of RS and FZ were finally retained. Besides, there are 3 common components (CC) shared by RS and FZ (Table 1).

Table 1. Active components of RS and FZ

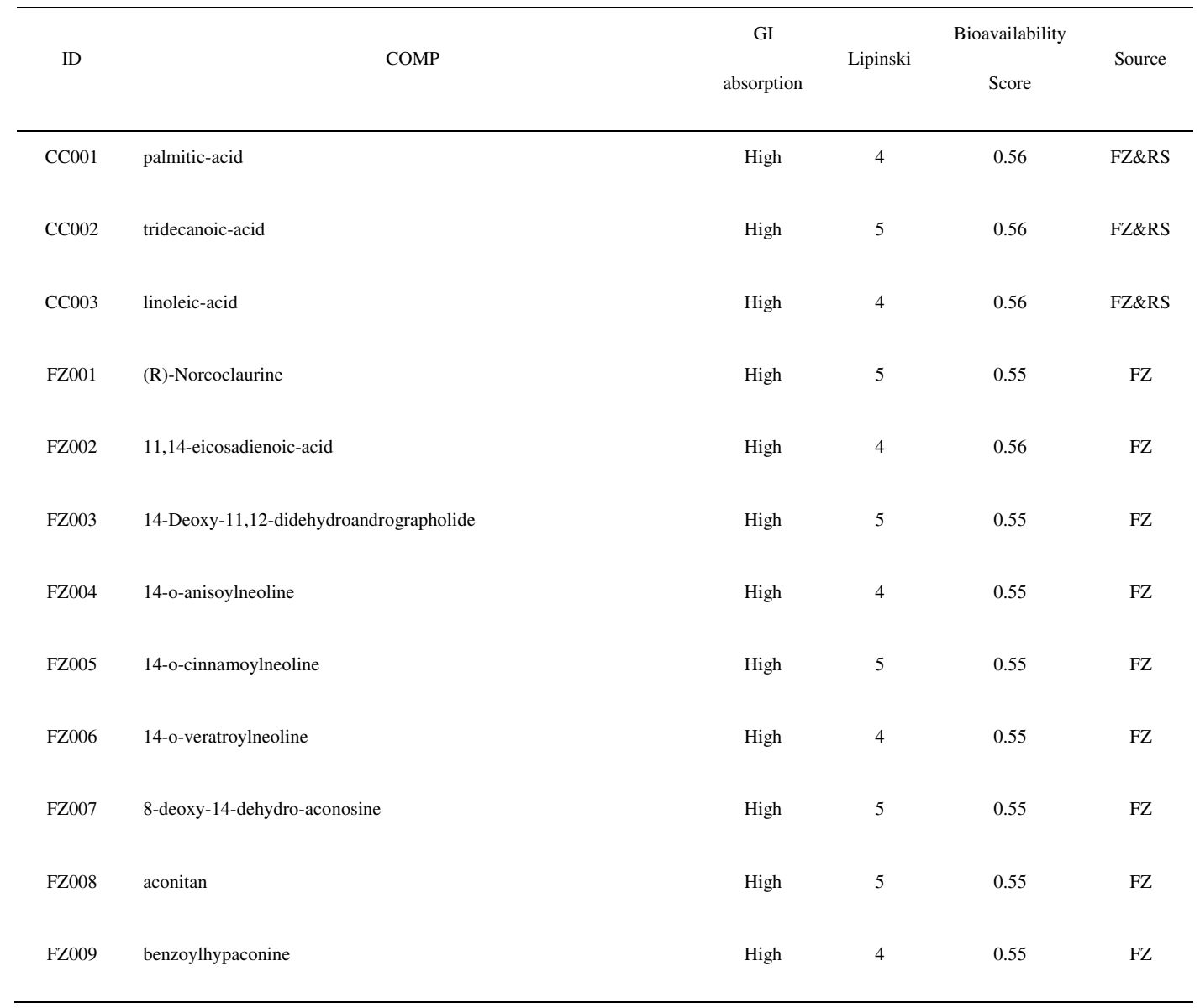




\begin{tabular}{|c|c|c|c|c|c|}
\hline FZ010 & benzoylnapelline & High & 5 & 0.55 & FZ \\
\hline FZ011 & bullatine-b & High & 5 & 0.55 & FZ \\
\hline FZ012 & bullatine-c & High & 5 & 0.55 & FZ \\
\hline FZ013 & carmichaeline & High & 5 & 0.55 & FZ \\
\hline FZ014 & chasmaconitine & High & 4 & 0.55 & $\mathrm{FZ}$ \\
\hline FZ015 & chasmanine & High & 5 & 0.55 & $\mathrm{FZ}$ \\
\hline FZ016 & coryneine & High & 5 & 0.55 & FZ \\
\hline FZ017 & delamide & High & 5 & 0.55 & $\mathrm{FZ}$ \\
\hline FZ018 & Delavaconitine & High & 5 & 0.55 & $\mathrm{FZ}$ \\
\hline FZ019 & Delbruline & High & 5 & 0.55 & $\mathrm{FZ}$ \\
\hline FZ020 & delbrunine & High & 5 & 0.55 & $\mathrm{FZ}$ \\
\hline FZ021 & Delbrusine & High & 5 & 0.55 & $\mathrm{FZ}$ \\
\hline FZ022 & Delcorine & High & 5 & 0.55 & $\mathrm{FZ}$ \\
\hline FZ023 & Delphamine & High & 5 & 0.55 & $\mathrm{FZ}$ \\
\hline FZ024 & delphatine & High & 5 & 0.55 & $\mathrm{FZ}$ \\
\hline FZ025 & Delsoline & High & 5 & 0.55 & $\mathrm{FZ}$ \\
\hline FZ026 & deltaline & High & 4 & 0.55 & $\mathrm{FZ}$ \\
\hline FZ027 & Deltamine & High & 5 & 0.55 & $\mathrm{FZ}$ \\
\hline FZ028 & Deltoin & High & 5 & 0.55 & FZ \\
\hline FZ029 & denudatine & High & 5 & 0.55 & $\mathrm{FZ}$ \\
\hline FZ030 & Deoxyandrographolide & High & 5 & 0.55 & $\mathrm{FZ}$ \\
\hline FZ031 & dictysine & High & 5 & 0.55 & $\mathrm{FZ}$ \\
\hline FZ032 & fuzitine & High & 5 & 0.55 & $\mathrm{FZ}$ \\
\hline FZ033 & glyceryl-monopalmitate & High & 5 & 0.55 & $\mathrm{FZ}$ \\
\hline FZ034 & hetisine & High & 5 & 0.55 & $\mathrm{FZ}$ \\
\hline FZ035 & higenamine & High & 5 & 0.55 & $\mathrm{FZ}$ \\
\hline FZ036 & hypaconine & High & 5 & 0.55 & $\mathrm{FZ}$ \\
\hline FZ037 & hypaphorine & High & 5 & 0.55 & FZ \\
\hline
\end{tabular}




\begin{tabular}{|c|c|c|c|c|c|}
\hline FZ038 & ignavine & High & 5 & 0.55 & $\mathrm{FZ}$ \\
\hline FZ039 & isodelphinine & High & 4 & 0.55 & $\mathrm{FZ}$ \\
\hline FZ040 & isotalatizidine & High & 5 & 0.55 & $\mathrm{FZ}$ \\
\hline FZ041 & karacoline & High & 5 & 0.55 & $\mathrm{FZ}$ \\
\hline FZ042 & Karanjin & High & 5 & 0.55 & $\mathrm{FZ}$ \\
\hline FZ043 & m-aminophenol & High & 5 & 0.55 & $\mathrm{FZ}$ \\
\hline FZ044 & mescaline & High & 5 & 0.55 & $\mathrm{FZ}$ \\
\hline FZ045 & myristic-acid & High & 5 & 0.56 & $\mathrm{FZ}$ \\
\hline FZ046 & Neojiangyouaconitine & High & 4 & 0.55 & $\mathrm{FZ}$ \\
\hline FZ047 & neojianyouaconitine & High & 4 & 0.55 & $\mathrm{FZ}$ \\
\hline FZ048 & o-aminophenol & High & 5 & 0.55 & $\mathrm{FZ}$ \\
\hline FZ049 & p-aminophenol & High & 5 & 0.55 & $\mathrm{FZ}$ \\
\hline FZ050 & penduline & High & 4 & 0.55 & $\mathrm{FZ}$ \\
\hline FZ051 & Pyrrolezanthine & High & 5 & 0.55 & $\mathrm{FZ}$ \\
\hline FZ052 & Salsolinol & High & 5 & 0.55 & $\mathrm{FZ}$ \\
\hline FZ053 & Senbusine-A & High & 5 & 0.55 & $\mathrm{FZ}$ \\
\hline FZ054 & Senbusine-B & High & 5 & 0.55 & $\mathrm{FZ}$ \\
\hline FZ055 & Senbusine-C & High & 5 & 0.55 & $\mathrm{FZ}$ \\
\hline FZ056 & songoramine & High & 5 & 0.55 & $\mathrm{FZ}$ \\
\hline FZ057 & songorine & High & 5 & 0.55 & $\mathrm{FZ}$ \\
\hline FZ058 & talatisamine & High & 5 & 0.55 & $\mathrm{FZ}$ \\
\hline FZ059 & talatizamine & High & 5 & 0.55 & $\mathrm{FZ}$ \\
\hline FZ060 & uracil & High & 5 & 0.55 & $\mathrm{FZ}$ \\
\hline FZ061 & urical & High & 5 & 0.56 & $\mathrm{FZ}$ \\
\hline FZ062 & vilmorrianine-C & High & 4 & 0.55 & $\mathrm{FZ}$ \\
\hline RS001 & (+)-Maalioxide & High & 5 & 0.55 & RS \\
\hline RS002 & $\begin{array}{l}(1 \mathrm{R}, 4 \mathrm{E}, 7 \mathrm{E}, 11 \mathrm{R})-1,5,9,9-\text { tetramethyl-12-oxabicyclo[9.1.0]dodeca-4,7- } \\
\text { diene }\end{array}$ & High & 5 & 0.55 & RS \\
\hline
\end{tabular}


(3R,5R,8R,9R,10R,12R,13R,14R,17S)-17-[(2S)-2-hydroxy-6-

methylhept-5-en-2-yl]-4,4,8,10,14-pentamethyl-

RS003

2,3,5,6,7,9,11,12,13,15,16,17-dodecahydro-1H-

cyclopenta[a]phenanthrene-3,12-diol

(3S,5R,6S,8R,9R,10R,12R,13R,14R,17S)-17-[(2S)-2-hydroxy-6-

RS004

methylhept-5-en-2-yl]-4,4,8,10,14-pentamethyl-

2,3,5,6,7,9,11,12,13,15,16,17-dodecahydro-1H-

cyclopenta[a]phenanthrene-3,6,12-triol

(3S,5R,8R,9R,10R,12R,13R,14R,17S)-17-[(2S)-2-hydroxy-6-

methylhept-5-en-2-yl]-4,4,8,10,14-pentamethyl-

RS005

2,3,5,6,7,9,11,12,13,15,16,17-dodecahydro-1H-

cyclopenta[a]phenanthrene-3,12-diol

RS006 (9R,10S)-Epoxyheptadecan-4,6-Diyn-3-One

RS007

(R)-(+)-Citronellal

RS008

12-O-Nicotinoylisolineolone

RS009

13-Tetradecenyl-acetate

RS010 16-Oxoseratenedio

RS011 1-heptadecanol

RS012 1-methoxy-(9r,10s)-epoxyheptadecan-4,6-diyn-3-one

RS013 1-tetradecanol

RS014 2,5-dimethyl-7-hydroxy-chromone

RS015 2,6-dimethyl-3,7-octadiene-2,6-diol

RS016 2,6-ditertbutyl-4-methyl-phenol

RS017 2-heptadecanone

RS018 3,5-dimethyl-4-methoxybenzoic-acid

RS019 3-[[(2S)-2,4-dihydroxy-3,3-dimethylbutanoyl]amino]propanoic-acid

RS020 3-Hydroxycoumarin,Folic-Acid

5-[(3aS,6R,6aR)-2-keto-1,3,3a,4,6,6a-hexahydrothieno[3,4

RS021

d]imidazol-6-yl]valeric-acid

RS022 9-HEXADECENOIC-ACID

RS023
High 


\begin{tabular}{|c|c|c|c|c|c|}
\hline RS024 & adenine & High & 5 & 0.55 & RS \\
\hline RS025 & alpha-cadinol & High & 5 & 0.55 & RS \\
\hline RS026 & alpha-cedrol & High & 5 & 0.55 & RS \\
\hline RS027 & alpha-santalol & High & 5 & 0.55 & RS \\
\hline RS028 & aposcopolamine & High & 5 & 0.55 & RS \\
\hline RS029 & Aposiopolamine & High & 5 & 0.55 & RS \\
\hline RS030 & arachidonate & High & 4 & 0.56 & RS \\
\hline RS031 & beta-santalol & High & 5 & 0.55 & RS \\
\hline RS032 & biotin & High & 5 & 0.56 & RS \\
\hline $\mathrm{RS} 033$ & Celabenzine & High & 5 & 0.55 & RS \\
\hline RS034 & chloropanaxydiol & High & 5 & 0.55 & RS \\
\hline RS035 & Chromone & High & 5 & 0.55 & RS \\
\hline RS036 & citronellal & High & 5 & 0.55 & RS \\
\hline RS037 & darutoside & High & 4 & 0.55 & RS \\
\hline RS038 & dauricine & High & 4 & 0.55 & RS \\
\hline RS039 & deoxygomisin-a & High & 5 & 0.55 & RS \\
\hline RS040 & Deoxyharringtonine & High & 4 & 0.55 & RS \\
\hline RS041 & Dianthramine & High & 5 & 0.56 & RS \\
\hline RS042 & dibutyl-oxalate & High & 5 & 0.55 & RS \\
\hline RS043 & dibutyl-phthalate & High & 5 & 0.55 & RS \\
\hline RS044 & Diop & High & 4 & 0.55 & RS \\
\hline RS045 & Ditertbutyl-phthalate & High & 5 & 0.55 & RS \\
\hline RS046 & dodecanol & High & 5 & 0.55 & RS \\
\hline RS047 & Elemicin & High & 5 & 0.55 & RS \\
\hline RS048 & Frutinone-A & High & 5 & 0.55 & RS \\
\hline RS049 & ginsenol & High & 5 & 0.55 & RS \\
\hline RS050 & Ginsenoyne-A & High & 5 & 0.55 & RS \\
\hline RS051 & Ginsenoyne-B & High & 5 & 0.55 & RS \\
\hline
\end{tabular}




\begin{tabular}{|c|c|c|c|c|c|}
\hline RS052 & Ginsenoyne-C & High & 5 & 0.55 & RS \\
\hline RS053 & Ginsenoyne-D & High & 5 & 0.55 & RS \\
\hline RS054 & Ginsenoyne-E & High & 5 & 0.55 & RS \\
\hline RS055 & girinimbine & High & 5 & 0.55 & RS \\
\hline RS056 & gomisin-a & High & 5 & 0.55 & RS \\
\hline RS057 & Gomisin-B & High & 4 & 0.55 & RS \\
\hline RS058 & humulene-epoxide-i & High & 5 & 0.55 & RS \\
\hline RS059 & Inermin & High & 5 & 0.55 & RS \\
\hline RS060 & isocnidilide & High & 5 & 0.55 & RS \\
\hline RS061 & kaempferol & High & 5 & 0.55 & RS \\
\hline RS062 & Linoleic & High & 4 & 0.56 & RS \\
\hline RS063 & Malic-Acid & High & 5 & 0.56 & RS \\
\hline RS064 & malkangunin & High & 5 & 0.55 & RS \\
\hline RS065 & Malvic-acid & High & 4 & 0.56 & RS \\
\hline RS066 & menthyl-acetate & High & 5 & 0.55 & RS \\
\hline RS067 & METHYL-LINOLEATE & High & 4 & 0.55 & RS \\
\hline RS068 & Methyl-margarate & High & 4 & 0.55 & RS \\
\hline RS069 & Methyl-myristate & High & 5 & 0.55 & RS \\
\hline RS070 & methyl-palmitate & High & 4 & 0.55 & RS \\
\hline RS071 & Methyl-palmitelaidate & High & 4 & 0.55 & RS \\
\hline RS072 & METHYL-PENTADECANOATE & High & 4 & 0.55 & RS \\
\hline RS073 & Methyl-stearate & High & 4 & 0.55 & RS \\
\hline RS074 & Mycosinol & High & 5 & 0.55 & RS \\
\hline RS075 & neointermedeol & High & 5 & 0.55 & RS \\
\hline RS076 & Nepetin & High & 5 & 0.55 & RS \\
\hline RS077 & Nicotinic-Acid & High & 5 & 0.56 & RS \\
\hline RS078 & NN-Dimethyldecanamide & High & 5 & 0.55 & RS \\
\hline RS079 & octanal & High & 5 & 0.55 & RS \\
\hline
\end{tabular}




\begin{tabular}{|c|c|c|c|c|c|}
\hline RS080 & paeonol & High & 5 & 0.55 & RS \\
\hline RS081 & palmitin & High & 5 & 0.55 & RS \\
\hline RS082 & palmitoleic-acid & High & 5 & 0.56 & RS \\
\hline RS083 & panasinsanol-a & High & 5 & 0.55 & RS \\
\hline RS084 & panasinsanol-b & High & 5 & 0.55 & RS \\
\hline RS085 & panaxacol & High & 5 & 0.55 & RS \\
\hline RS086 & Panaxadiol & High & 4 & 0.55 & RS \\
\hline RS087 & panaxatriol & High & 4 & 0.55 & RS \\
\hline RS088 & panaxydol & High & 5 & 0.55 & RS \\
\hline RS089 & panaxynol & High & 4 & 0.55 & RS \\
\hline RS090 & Panaxytriol & High & 5 & 0.55 & RS \\
\hline RS091 & Pandamine & High & 4 & 0.55 & RS \\
\hline RS092 & patchouli-alcohol & High & 5 & 0.55 & RS \\
\hline RS093 & pentadecanoic-acid & High & 5 & 0.56 & RS \\
\hline RS094 & protopanaxadiol & High & 4 & 0.55 & RS \\
\hline RS095 & protopanaxatriol & High & 4 & 0.55 & RS \\
\hline RS096 & protopanaxtriol & High & 4 & 0.55 & RS \\
\hline RS097 & protopine & High & 5 & 0.55 & RS \\
\hline RS098 & putrescine & High & 5 & 0.55 & RS \\
\hline RS099 & pyrrole-2-aldehyde & High & 5 & 0.55 & RS \\
\hline RS100 & Pyruvic-Acid & High & 5 & 0.56 & RS \\
\hline RS101 & Ramalic-acid & High & 5 & 0.56 & RS \\
\hline RS102 & rhamnose & High & 5 & 0.55 & RS \\
\hline RS103 & Salicylic-acid & High & 5 & 0.56 & RS \\
\hline RS104 & se-methyl-1-selenocysteine & High & 5 & 0.55 & RS \\
\hline RS105 & spermidine & High & 5 & 0.55 & RS \\
\hline RS106 & spermine & High & 5 & 0.55 & RS \\
\hline RS107 & stigmasterol-beta-d-glucoside & High & 4 & 0.55 & RS \\
\hline
\end{tabular}




\begin{tabular}{|c|c|c|c|c|c|}
\hline RS108 & Succinic-Acid & High & 5 & 0.56 & RS \\
\hline RS109 & suchilactone & High & 5 & 0.55 & RS \\
\hline RS110 & trans-9-trans-12-linoleic-acid & High & 4 & 0.56 & RS \\
\hline RS111 & Trifolirhizin & High & 5 & 0.55 & RS \\
\hline RS112 & vitamin-b5 & High & 5 & 0.56 & RS \\
\hline RS113 & Vulgarin & High & 5 & 0.55 & RS \\
\hline RS114 & widdrol & High & 5 & 0.55 & RS \\
\hline RS115 & Ginsenoside-f1 & Low & 3 & 0.17 & RS \\
\hline RS116 & Ginsenoside-f4 & Low & 2 & 0.17 & RS \\
\hline RS117 & Ginsenoside-I & Low & 2 & 0.17 & RS \\
\hline RS118 & Ginsenoside-Ia & Low & 2 & 0.17 & RS \\
\hline RS119 & Ginsenoside-II & Low & 2 & 0.17 & RS \\
\hline RS120 & Ginsenoside-III & Low & 2 & 0.17 & RS \\
\hline RS121 & Ginsenoside-La & Low & 2 & 0.17 & RS \\
\hline RS122 & Ginsenoside-Ra0 & Low & 2 & 0.17 & RS \\
\hline RS123 & Ginsenoside-Ra1 & Low & 2 & 0.17 & RS \\
\hline RS124 & Ginsenoside-Ra2 & Low & 2 & 0.17 & RS \\
\hline RS125 & Ginsenoside-Ra3 & Low & 2 & 0.17 & RS \\
\hline RS126 & Ginsenoside-Rb1 & Low & 2 & 0.17 & RS \\
\hline RS127 & Ginsenoside-Rb2 & Low & 2 & 0.17 & RS \\
\hline RS128 & Ginsenoside-Rb3 & Low & 2 & 0.17 & RS \\
\hline RS129 & Ginsenoside-Rc & Low & 2 & 0.17 & RS \\
\hline RS130 & Ginsenoside-Rd & Low & 2 & 0.17 & RS \\
\hline RS131 & Ginsenoside-Re & Low & 2 & 0.17 & RS \\
\hline RS132 & Ginsenoside-Rf & Low & 2 & 0.17 & RS \\
\hline RS133 & Ginsenoside-Rg1 & Low & 2 & 0.17 & RS \\
\hline RS134 & Ginsenoside-Rg2 & Low & 2 & 0.17 & RS \\
\hline RS135 & Ginsenoside-Rg3 & Low & 2 & 0.17 & RS \\
\hline
\end{tabular}




\begin{tabular}{llllll}
\hline RS136 & Ginsenoside-Rg5 & Low & 2 & 0.17 & RS \\
RS137 & Ginsenoside-Rh1 & Low & 3 & 0.17 & RS \\
RS138 & Ginsenoside-Rh2 & Low & 3 & 0.17 & RS \\
RS139 & Ginsenoside-Rh3 & Low & 4 & 0.55 & RS \\
RS140 & Ginsenoside-Rh4 & Low & 3 & 0.17 & RS \\
RS141 & Ginsenoside-Ro & Low & 2 & 0.11 & RS \\
RS142 & Ginsenoside-Ro_qt & Low & 4 & 0.56 & RS \\
RS143 & Ginsenoside-Rs1 & Low & 2 & 0.17 & RS \\
RS144 & Ginsenoside-Rs2 & Low & 2 & 0.17 & RS \\
\hline
\end{tabular}

\subsubsection{Potential active components from RS}

After ADME screening, totaling 147 potential active components of RS were obtained, including 30 ginsenosides that are important but have been screened out. Modern pharmacological researches show that the main active components of RS are ginsenosides, which are reported to have functions of anti-apoptosis and promoting vascular endothelial growth [29,30]. For example, Ginsenoside Re (RS131) and ginsenoside Rb1(RS126) can inhibit the apoptosis of cardiomyocytes by reducing the expression of pro-apoptotic genes Bax, Bad and Fas [31]. Similarly, Ginsenoside $\mathrm{Rh} 3$ (RS139) can also reduce cardiomyocytes apoptosis by increasing the expression of Bcl-2, while inhibiting the expression of Bax and Caspase-3 in myocardial tissue [32]. Besides, Ginsenoside Rg1 (RS133) has a significant angiogenic effect according to published reports. It is reported that Ginsenoside $\operatorname{Rg} 1$ can arouse sustained vascular regeneration by increasing the expressions of hypoxia inducible factor $-1 \alpha$, vascular endothelial growth factor and vascular endothelial growth factor receptor [33]. Additionally, Paeonol (RS080) can also protect cardiomyocytes and inhibit apoptosis of cardiomyocytes by inhibiting PI3K/AKT/mTOR and NF-kB signaling pathways [34].

\subsubsection{Potential active components from FZ}

Through ADME screening, 65 active components of FZ have been left for following analysis. Pharmacological studies show that FZ has the functions of strengthening heart and protecting myocardium. The active components of FZ mainly include alkaloids, aconite polysaccharides, amides, etc. For instance, Higenamine (FZ035) is an adrenergic receptor agonist, which exerts a cardiotonic effect by stimulating $\beta$-adrenergic receptor to produce positive force and timing effects, increasing the contraction frequency and amplitude of heart. Its effect on left ventricular function is similar to that of isoproterenol, or even be superior to isoproterenol on the treatment of bradycardia [35]. Coryneine (FZ016) is a derivative of dopamine, which has obvious boosting and cardiac strengthening effects and is able to increase the contraction amplitude and frequency of atria [36]. Salsolinol (FZ052) is a weak $\beta$-adrenergic stimulant. Animal experiments show that Salsolinol can stimulate isolated guinea pig atria and increase its contraction frequency [37]. Uracil (FZ060) and 
hypaconine (FZ036) have obvious cardiac activity and can significantly increase cardiac contractility and cardiac output, but have no effects on heart rate [38].

\subsection{Target prediction and analysis of RS-FZ}

By using the three online tools, SwissTargetPrediction, HITPICK and SEA, we have totally obtained 1030 targets corresponding to 147 active components of RS and 599 targets corresponding to 65 active components of FZ. Otherwise, the 3 common components of RS and FZ have 150 predicted targets. After data integration, 1210 targets corresponding to 209 potential active components of SFD were retrieved.

In order to dissect the mechanisms of SFD in the treatment of SSS, 209 active components and 1210 targets were used to construct the C-T network (Table S3, Fig. 3).

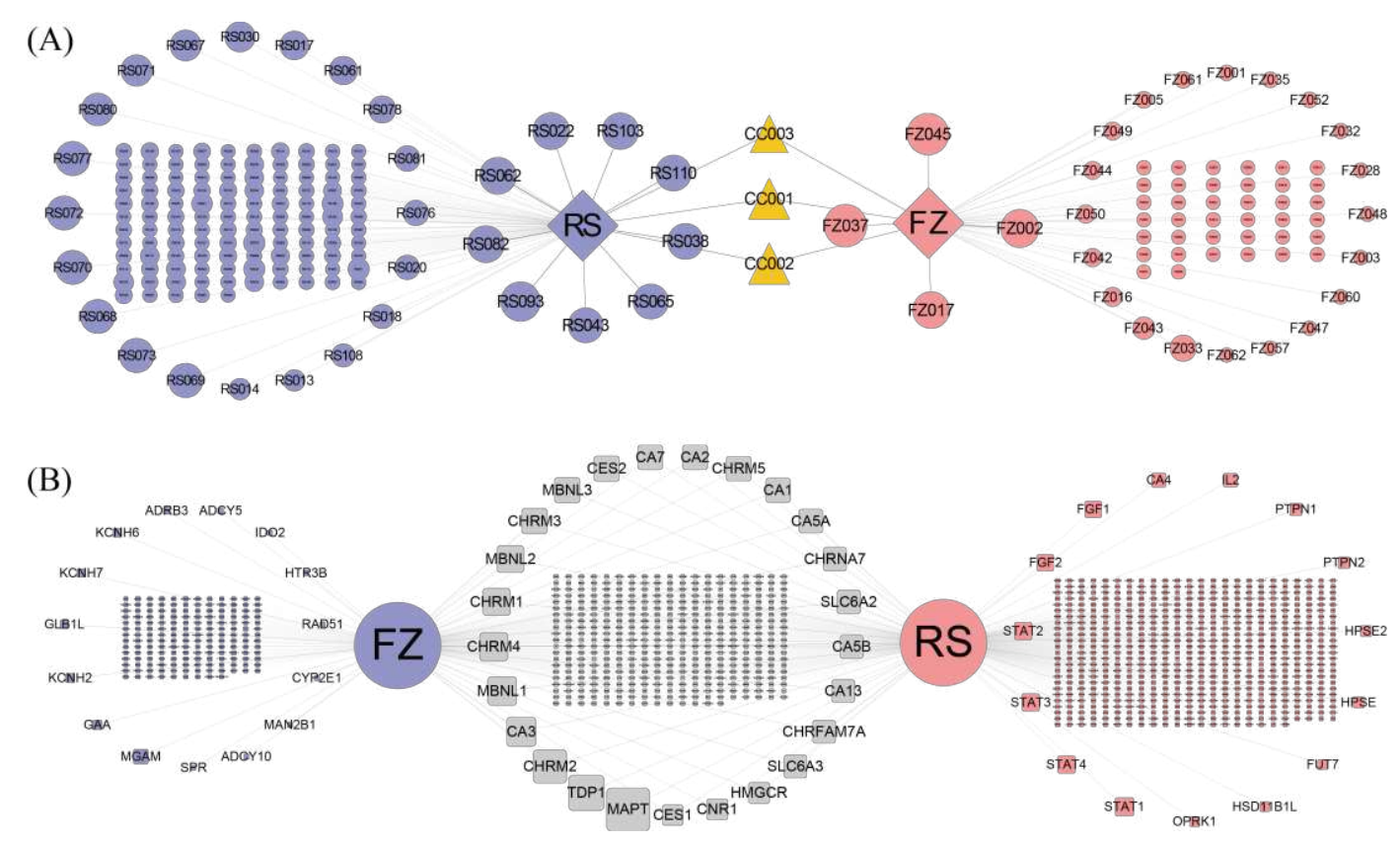

Fig. 3. Components-Targets network of SFD. (A)Herbs-Components network. The node size means the number of genes that are targeted by the node. The blue nodes belong to RS, the pink nodes belong to FZ while the orange nodes are the common components of RS and FZ. (B)HerbsTargets network. The node size represents the number of components target to the node. The blue nodes belong to FZ, the pink nodes belong to RS while the grey nodes are the common targets of RS and FZ.

There are 6978 component-target associations constituted by 209 active components and 1210 predicted targets in C-T network. Each active component corresponds to 33.39 targets on average, while the average number of active components corresponding to each target is 5.77 , which indicates that SFD has multi-component and multi-target characteristics in treating SSS

Among all the active components, 16 components had the number of degrees greater or equal to 100. The top of these components is Hypaphorine (FZ037, degree=132), followed by tridecanoic-acid $(\mathrm{CC} 002$, degree $=128)$ and myristic-acid $($ FZ045, degree $=128)$. Palmitic-acid 
(CC001, degree=127), pentadecanoic-acid (RS093, degree=119), linoleic-acid (CC003, degree=113), 11,14-eicosadienoic-acid (FZ002, degree=113), delamide (FZ017, degree=108), 9HEXADECENOIC-ACID (RS022, degree=108), Linoleic (RS062, degree=108), palmitoleic-acid (RS082, degree=108), Salicylic-acid (RS103, degree=107), dauricine (RS038, degree=104), trans9-trans-12-linoleic-acid (RS110, degree=104), Malvic-acid (RS065, degree=101) and dibutylphthalate (RS043, degree $=100$ ) are behind them. These results suggest that these components with high degrees may play fundamental roles in the treatment of SSS by SFD.

In RS, 147 active components correspond to 1030 predicted targets, constituting 5364 component-target interactions. To clarify the mechanism of RS, we constructed a C-T network with components and targets of RS. Through topological analysis of the C-T network, we found that CC002 (Degree=128) had the highest number of degrees among all RS active components, while CC001 (Degree=127) and RS093 (Degree=119) were followed. For the targets, the degrees of MAPT, MBNL1 and CA3 were the highest, which are 68, 44 and 43 respectively. MAPT encodes the microtubule-associated protein tau. Study has shown that tau deficient mice has lower right atrial rate and decreased inotropic responses to calcium, isoprenaline, and electrical sympathetic nerve stimulation with age [39]. MBNL1 can directly regulate the alternative splicing of exon $6 \mathrm{~A} / 6 \mathrm{~B}$ of SCN5A, which leads to delayed cardiac conduction and various arrhythmias [40].

In FZ, 599 targets are corresponded to 65 active components with 1982 interactions constituted among them. Similarly, we constructed a C-T network to analyze the mechanism of FZ. FZ037 (Degree=132), CC002 (Degree=128) and FZ045 (Degree=128) are found to have top three degrees among 65 active components of FZ, while CHRNA7 (Degree=39), CHRFAM7A (Degree=38) and CHRM2 (Degree=34) are three targets with top degrees among all the targets of FZ. CHRNA7 encodes cholinergic receptor nicotinic alpha 7 subunit, which is involved in the activation of cholinergic anti-inflammatory pathway in vivo. It can reduce the production of inflammatory cytokines by enhancing vagus nerve activity, so as to reduce tissue damage and has important cardiac protective effect [41]. CHRM2 encodes cholinergic receptor M2, which is mainly distributed in sinoatrial node, atrium, atrioventricular node and ventricle. It is involved in activating $\mathrm{K}^{+}$channel or inhibiting $\mathrm{Ca}^{2+}$ channel, and plays an important role in heart rate regulation.

\subsection{SIC and TCO calculation and effect verification}

From the above results, it can be found that there are many potentially active components in SFD, but preliminary network analysis suggests that each component plays different roles in the treatment of SSS. In order to explore the synergistic role of active components in SFD and understand the importance of each active component in disease treatment, we established a mathematical model based on C-T network to calculate the SIC and TCO values of SFD components in treating SSS (Table S4). 75 components with the accumulated SIC more than 90\% were defined as group of key synergistic components, while 75 components with accumulated TCO more than $90 \%$ were defined as group of high coverage components. Components with 
higher SIC score and TCO score could reflect the synergism and importance. Thus, we selected the 55 components which shared by group of key synergistic components and group of high coverage components and defined as SFCG (Fig. 4).

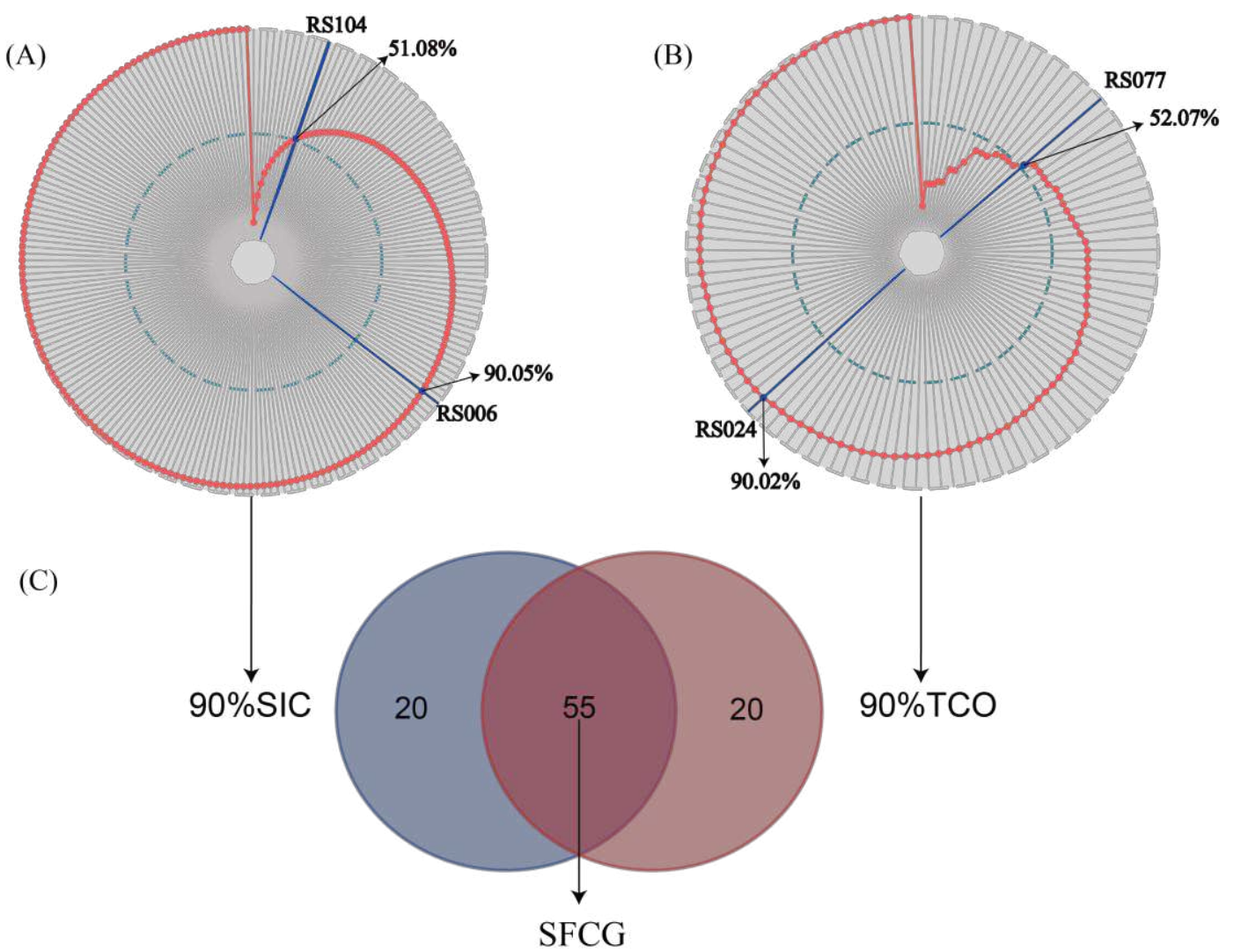

Fig. 4. The calculation result of SIC and TCO. (A)The calculation result of SIC. (B) The calculation result of TCO. (C)The selected SFCG with 55 components.

It is surprising that 55 components only account for $26.32 \%$ (55/209) of all active components in SFD and corresponds to 995 targets, which cover $82.23 \%$ of all SFD targets. These results show that SFCG play key roles in all the active components of SFD and may clarify why the herbs in SFD could generate synergistic and combination effects on SSS.

Among the 55 components of SFCG, 14 components belong to FZ and 43 components belong to RS. In addition, the 14 components of FZ associate with 505 targets, and the 43 components of RS correspond to 813 targets, while there are 323 shared targets between them. And before the SFCG has been calculated, 65 active components of FZ correspond to 599 targets, and 147 components of RS correspond to 1030 targets, with a total of 419 identical targets. From this analysis, we can infer that RS and FZ may play a synergistic mechanism in SFD treating SSS. Additionally, topology analysis of the $\mathrm{C}-\mathrm{T}$ network shows that the average degree of common targets of RS and FZ is 12.13, while 1.56 and 2.64 are the average degrees of unique targets of RS and FZ respectively, which indicates that RS and FZ play a synergistic therapeutic effect in treating SSS with remarkable importance.

In order to evaluate the role of SFCG in treating SSS, we compared the GO biological 
process (BP) terms and KEGG pathway terms among 995 targets of SFCG, 1210 targets of SFD components and 347 targets with relevant score $\geqslant 13.65$ (the median value) of SSS. P.adjustvalues were set at 0.05 as the cut-off criterion to identify the biological significance of the primary target. 995 targets of SFCG were enriched in 1961 GO-BP terms and mapped on 174 KEGG pathways, 1210 targets of SFD components were enriched in 2414 GO-BP terms and mapped on 186 KEGG pathways, while 347 pathogenetic genes of SSS were enriched in 2857 GO-BP terms and mapped on 152 KEGG pathways. For further analysis, targets of SFD and pathogenetic genes of SSS shared 1613 enriched GO-BP terms and 120 KEGG pathways, which were defined as Reference GO-BP and Reference KEGG respectively. 1892 enriched GO-BP terms (1892/2414, $78.38 \%$ ) and 169 enriched KEGG pathways (169/186, 90.86\%) of SFCG targets were overlapped with the enriched GO-BP terms and KEGG pathways of SFD targets. This result suggested that our proposed SFCG selecting model is reliable and accurate at the functional level. Additionally, 1299 enriched GO-BP terms of SFCG targets covered 80.53\% (1299/1613) Reference GO-BP, while 111 enriched KEGG pathways of SFCG targets covered 92.50\% (111/120) Reference KEGG, which also confirmed the reliability and effectiveness of our strategy to find the SFCG (Fig. 5).
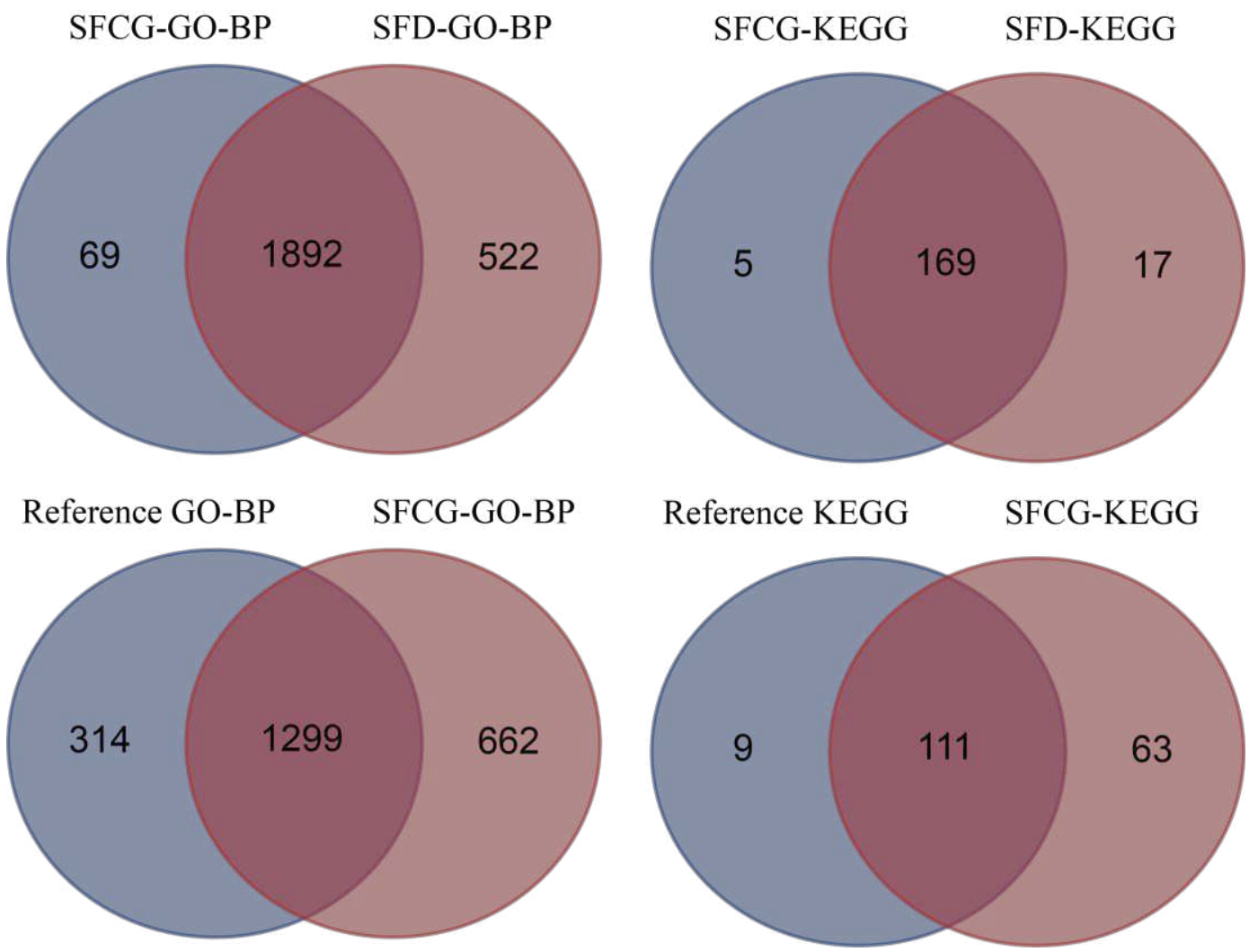

Fig. 5. Functional validation of SFCG in treating SSS.

3.5. Potential synergistic mechanisms analysis of SFD in treat of SSS based on HCGC

To fully understand the potential synergistic mechanism of RS and FZ, we used the targets of RS in SFCG and targets for FZ in SFCG to carry out the GO biological processes (BP) analysis 
and KEGG pathway analysis. P.adjust-values were set at 0.05 as the cut-off criterion to identify the biological significance of the primary targets.

3.5.1. GO enrichment analysis for SFD based on SFCG

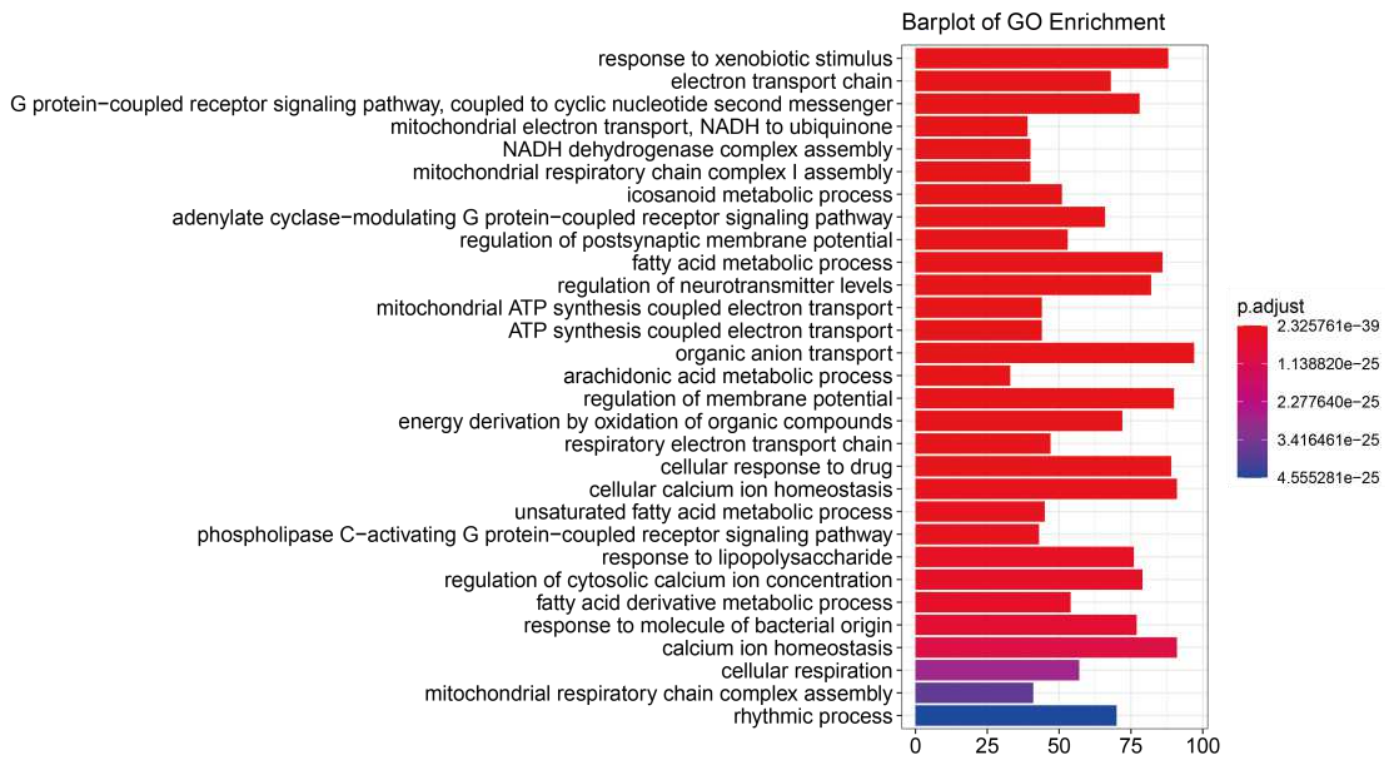

Fig. 6. The top 30 enriched GO-BP terms of SFCG targets.

GO enrichment analysis showed that targets of SFCG were enriched on the 1961 GO-BP terms and most of them are closely related to SSS. To our surprise, the top 30 GO-BP terms sort by P.adjust values from small to large mainly includes items related to calcium signal, mitochondria and signal transduction (Fig. 6). From many published researches we can know that calcium circulation in sinus node cells is an important material basis for cardiac pacing. The speed of calcium circulation is one of the most important determinants of regulating the cardiac pacing, which is of great significance to the regulation of heart rate [42]. Biological processes related to calcium circulation include regulation of cellular calcium ion homeostasis (GO:0006874, CACNA1A, CALM1, CACNA1D, CACNA2D1, etc.), regulation of cytosolic calcium ion concentration (GO:0051480, CAMK2D, CALM1, CACNA1D, RYR2, CACNA2D1, etc.), calcium ion homeostasis (GO:0055074, ADORA1, CAMK2D, CACNA1A, CALM1, CACNA1D, CACNA2D1, etc.). Besides that, the mitochondrial dysfunction is reported to lead to the imbalance of ion channels in myocardial cells, which will cause various arrhythmias [43]. Biological processes involving mitochondria include electron transport chain (GO:0022900, NDUFA1, NDUFA2, NDUFA3, NDUFA4, etc.), mitochondrial electron transport, NADH to ubiquinone (GO:0006120, NDUFA1, NDUFS1, NDUFV1, etc.), NADH dehydrogenase complex assembly (GO:0010257, NDUFA1, NDUFB1, NDUFC1, etc.), mitochondrial respiratory chain complex I assembly (GO:0032981, NDUFA1, NDUFB1, NDUFC1, etc.) and so on. In addition, G protein-coupled receptor can mediate many kinds of signal transduction on cardiomyocytes, such as cholinergic signal, adrenergic signal, calcium signal and so on [44], which are significant for heart rate regulation and cardiac conduction. The related biological processes include $\mathrm{G}$ protein-coupled receptor signaling pathway, coupled to cyclic nucleotide second messenger (GO:0007187, ADORA1, CHRM2, CACNA1D, etc.), 
adenylate cyclase-modulating $\mathrm{G}$ protein-coupled receptor signaling pathway (GO:0007188, ADORA1, ADRA2A, DRD2, etc.), phospholipase C-activating $G$ protein-coupled receptor signaling pathway (GO:0007200, ADRA2A, CHRM2, DRD2, etc.) and so on.

To make a further dissection of the potential synergistic mechanisms of RS and FZ, we did GO-BP enrichment analysis for targets of RS in SFCG and targets of FZ in SFCG respectively. Results showed that there are 1305 GO-BP items enriched by 505 FZ targets and 1754 GO-BP items enriched by 813 RS targets, with 1051 identical terms. Then we compare the upper quartile of GOBP terms of RS targets, FZ targets and SSS pathogenetic genes, which sort by P.adjust values from small to large to find the shared GO terms among them (Fig. 7). From the result we can find that there are 101 shared GO-BP terms related to SSS between targets of RS and FZ, including GO:0042391: regulation of membrane potential, GO:0006874: cellular calcium ion homeostasis, GO:0055074: calcium ion homeostasis, GO:0051480: regulation of cytosolic calcium ion concentration, GO:0007204: positive regulation of cytosolic calcium ion concentration and so on, which are closely related to ion channel function. And the dysfunction of ion channels can lead to abnormal automatic depolarization of sinoatrial node cell membrane, resulting in unusual cardiac pacing and conduction $[45,46]$.

From the analysis above, we can know that RS and FZ play a synergistic role in regulation of calcium signal, mitochondrial function and signal transduction in myocadiac cells, which can be considered as the biological process mechanisms of SFD in treating SSS.

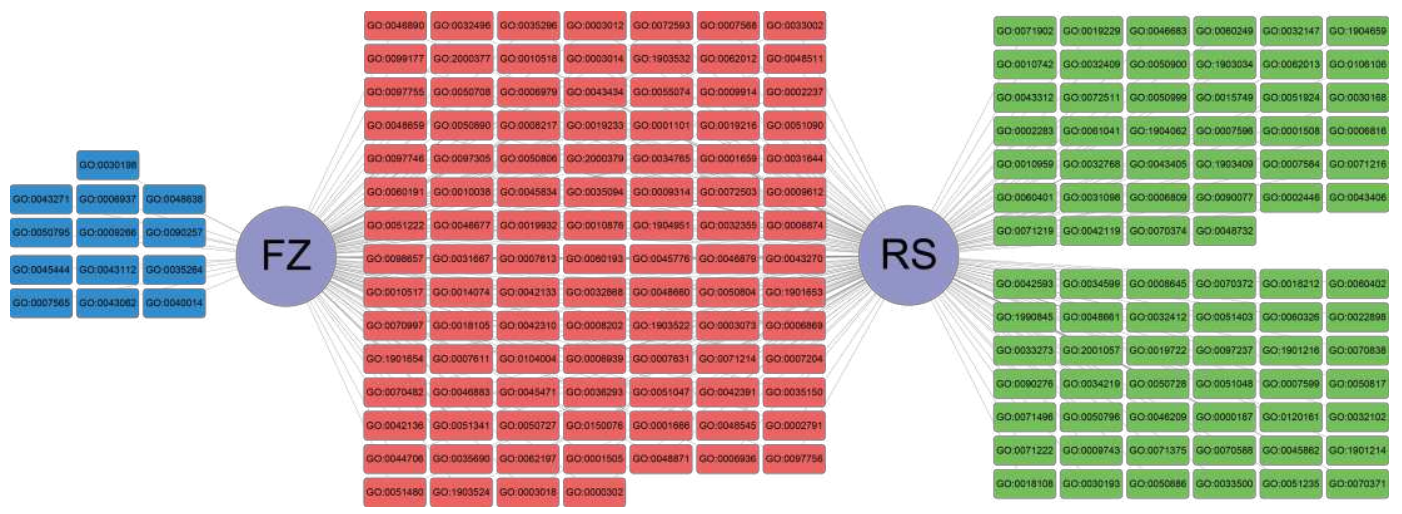

Fig. 7. The upper quartile of GO-BP terms of RS and FZ. The blue and green rectangular nodes are the unique GO-BP terms of FZ targets and RS targets respectively, while the red ones are the shared GO-BP terms of RS targets and FZ targets.

3.5.2. Pathway analysis to explore the potential synergistic mechanisms of SFD based on SFCG 


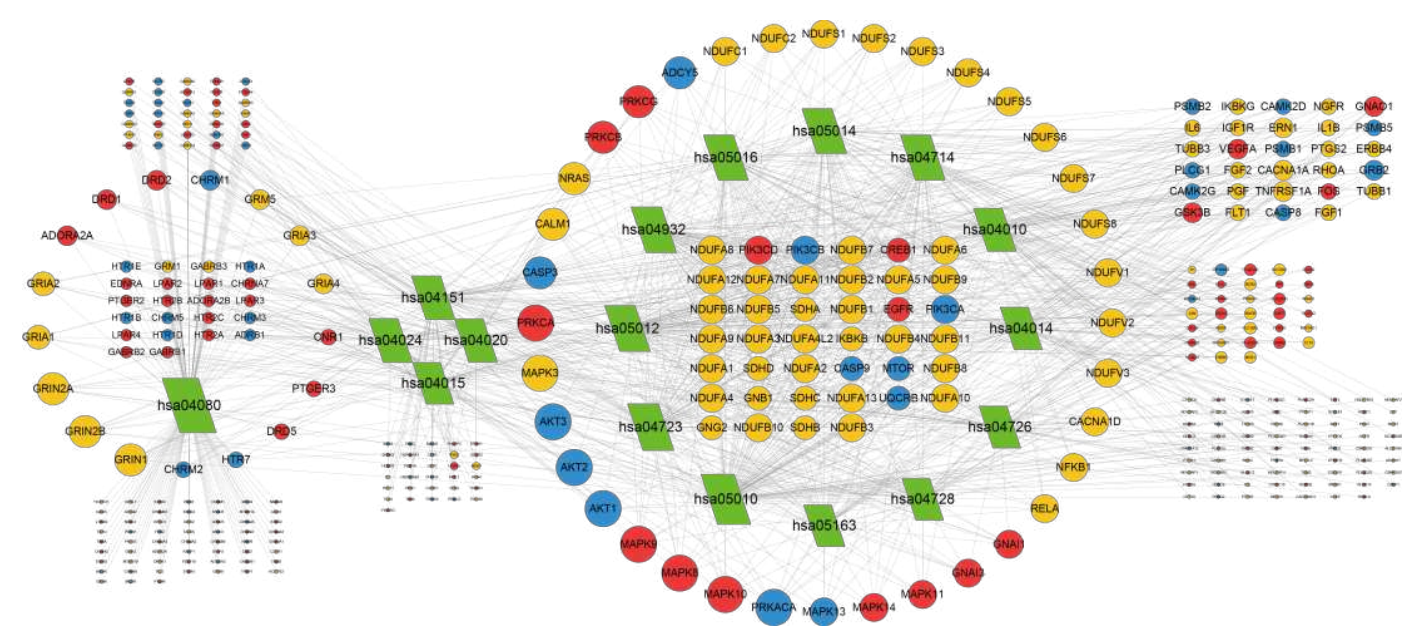

Fig. 8. Targets-Pathways network of SFD. The orange and blue nodes are the unique targets of RS and FZ respectively, while the red ones are the shared targets of RS and FZ. The green nodes are the pathways of targets of SFD.

To elaborate on the significant pathways involved in SFD for SSS therapy, targets of SFCG were mapped onto the KEGG pathways. Based on the mapping result, we constitute a targetspathways (T-P) network (Fig. 8) by using the top 10\% count of KEGG terms and their related targets. The T-P network contains 390 nodes (373 targets, 17 pathways, 1140 edges). The T-P network analysis result suggests that "multi-target" and "multi-pathway" are the characters within the SFD for SSS treatment. Based on the analysis result of the T-P network, PRKACA (Degree=10), AKT1 (Degree=9), AKT2 (Degree=9), AKT3 (Degree=9), MAPK10 (Degree=9), MAPK3 (Degree=9), MAPK8 (Degree=9), MAPK9 (Degree=9) and PRKCA (Degree=9) are the top 9 targets in the network. PRKACA encodes one of the catalytic subunits of protein kinase A, which is involved in the activation of Ryanodine Receptor 2 (RYR2) and regulation of calcium circulation in myocardial cells [47]. And the protein encoded by PRKCA is a member of PKC family, playing a role as fundamental regulator of contraction and calcium circulation in cardiomyocyte [48]. AKT1, AKT2 and AKT3 are three isoforms of Akt kinase and critical for cell survival, growth and proliferation of the cardiovascular system [49]. Additionally, MAPK3, MAPK8, MAPK9 and MAPK10 are members of MAP kinase family, which is reported to closely relate with cardiac development, ischemia and hypertrophy [50].

Among all the pathways, hsa04080: Neuroactive ligand-receptor interaction is the pathway that has most associated targets (Count=133), followed by hsa05010: Alzheimer disease (Count=103) and hsa04723: Retrograde endocannabinoid signaling (Count=80). The current knowledge believe that heart is dominated by sympathetic and parasympathetic nerves, they release adrenaline and acetylcholine respectively through their endings, which combine with receptors on the heart, thus regulating the contractility, rhythm and rate of the heart. In addition, according to published researched, cAMP signaling pathway, calcium signaling pathway, PI3K-Akt signaling pathway and Rap1 signaling pathway are closely related to the pathogenic mechanisms of SSS. Calcium plays an important role in the excitation-contraction coupling of myocardia and the regulation of action potential. When myocardial cells are depolarized, the calcium channel on the cell membrane opens, 
which leads to the entry of calcium into cells, and then triggers sarcoplasmic reticulum to release a large amount of calcium and combine with troponin, which leads to myofilament sliding and myocardial contraction [51]. PI3K signal can be involved in regulating the expression of sodium, potassium and calcium channels in cardiac myocytes, thus affecting the time course of cardiac action potential and playing a role in regulating heart rate and cardiac conduction [52]. Rap1 is a small GTP enzyme, which participates in many intracellular biochemical processes, and can induce calcium release during cardiac excitation and contraction. The low expression or deletion of Rap1 will lead to atrioventricular block, prolonged QRS duration, slow heart beat and decreased cardiac output [53]. Besides, cAMP is one of the most common second messengers, which responds to extracellular signals by changing its concentration, thus triggering a series of biochemical reactions in the cells, which are closely related to many signal pathways, including calcium signal pathway, PI3K-AKT signal pathway, Rap1 signal pathway and so on. Studies have shown that with the increase of age, the dysfunction of sinoatrial node cells gradually accumulates, and the reactivity to the activation of M-cholinergic receptor and adrenergic receptor decreases, which leads to the decrease of intracellular cAMP concentration, which leads to the disorder of calcium ion circulation in myocardial cells and slows down the heart rate [54]. The above results indicate that SFD may regulate the contractility, rhythm and beating rate of the heart by regulating the ion channel functions of the myocardia cells, which can be considered as the major mechanisms of SFD in treating SSS.

Similarly, to make a further exploration of the potential synergistic mechanisms of RS and FZ, we did KEGG enrichment analysis for targets of RS in SFCG and targets for FZ in SFCG respectively. The results show that there are 162 pathways enriched by $505 \mathrm{FZ}$ targets and 139 pathways enriched by 813 RS targets, with 117 shared pathways between them. In the comparison of the top $30 \mathrm{KEGG}$ entries of RS targets and FZ targets, there are 10 identical entries, which includes hsa04080: Neuroactive ligand-receptor interaction, hsa00590: Arachidonic acid metabolism, hsa04726: Serotonergic synapse, hsa04728: Dopaminergic synapse, hsa04071: Sphingolipid signaling pathway, hsa04931: Insulin resistance, hsa00591: Linoleic acid metabolism, hsa04933: AGE-RAGE signaling pathway in diabetic complications, hsa00910: Nitrogen metabolism, hsa04750: Inflammatory mediator regulation of TRP channels. This result, together with the GO-BP analysis and target analysis above, shows that RS and FZ in SFD have an important synergistic effect in treating SSS.

3.6. Comprehensive pathway analysis for SFD in treating SSS 


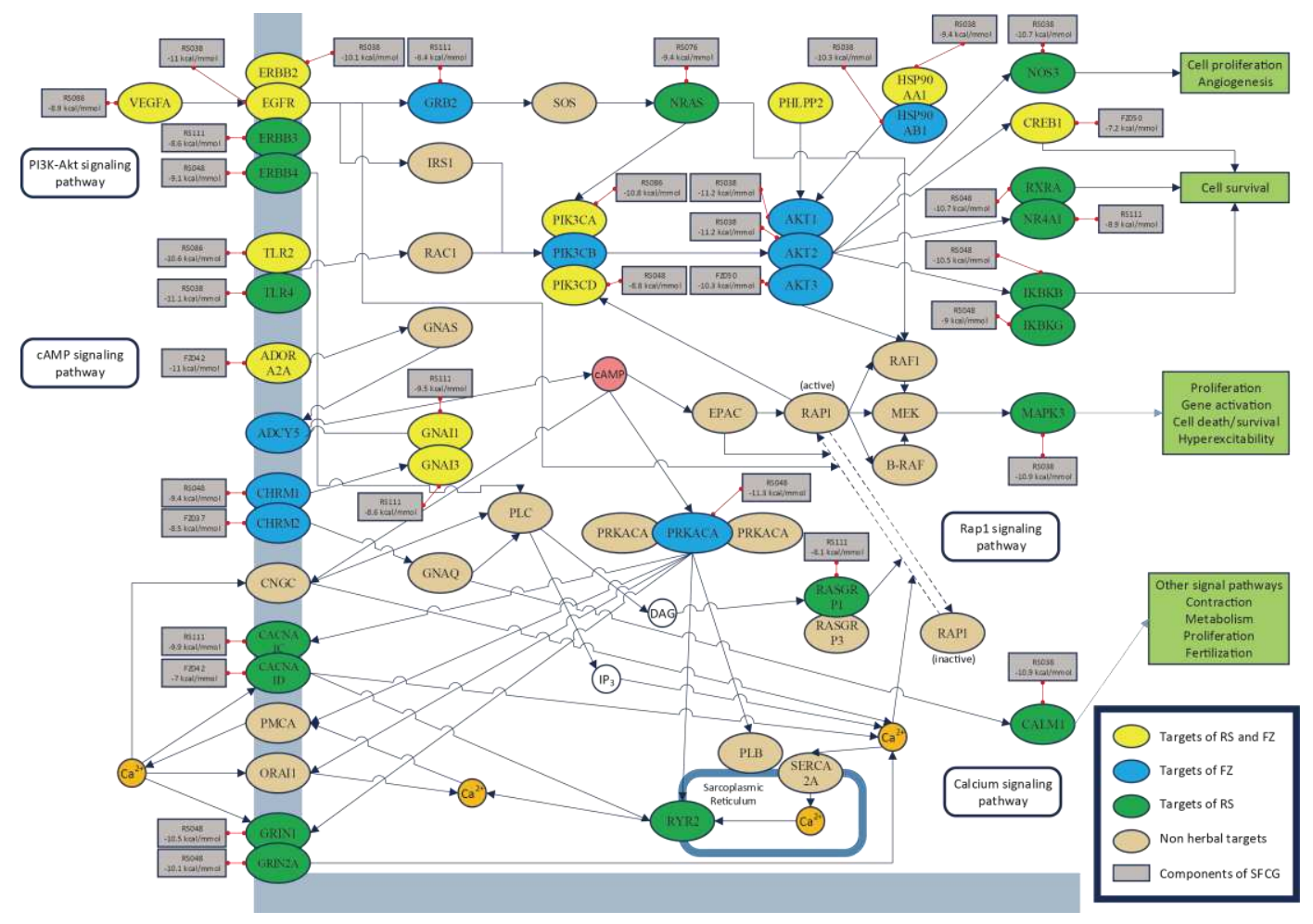

Fig. 9. The comprehensive pathway of SFD in treating SSS.

In order to further explore the synergistic mechanism of RS and FZ in the treatment of SSS in SFD, we constructed a comprehensive pathway. As shown in Fig. 9, the comprehensive pathway consists of cAMP, PI3K-Akt, calcium and Rap1 signals mainly focuses on cell proliferation, angiogenesis, cell survival, contraction and so on, which are closely related to SSS according to previous analysis. The pathway contains VEGFA, EGFR, GRB2, PI3K, AKT and NOS3 can regulate cell proliferation and angiogenesis. Studies showed that the overexpression of vascular endothelial growth factor could lead to irregular and tortuous capillaries of the sinus node, which make the sinus node smaller and less compact, leading to sinus node dysfunction and bradyarrhythmia [55]. Additionally, ADORA2A, ADCY5, PRKACA, CACNA1C, CACNA1D, RYR2, GRIN1 and CALM1 contribute to cAMP and calcium signaling pathway, which can regulate calcium circulation to handle the contraction of the heart. In the comprehensive pathway, the first three columns of targets are defined as upstream targets, while others are defined as downstream targets. According to Fig. 9, there are $46.15 \%$ (18/39) herbal targets acting on the upstream pathway, which contains $40 \%(4 / 10)$ particular targets of FZ and $41.18 \%(7 / 17)$ particular targets of RS. Besides, the particular targets of RS and shared targets of RS and FZ play major synergistic role in the upstream pathway while particular targets of RS and FZ in the downstream. All the targets mentioned above belong to RS or FZ and they play different roles in different parts of the pathways, which indicates the synergistic therapeutic effect of RS and FZ in the treatment of SSS with SFD.

\subsection{Molecular docking validation analysis}

In order to validate the synergistic effect of RS and FZ, 35 proteins including 11 proteins of RS and FZ, 8 of RS and 16 of FZ were conducted molecular docking with 55 components of SFCG, generating a total of 1925 docking results (Table S5), 99.38\% (1913/1925) of which have the 
affinity less than $0 \mathrm{kcal} / \mathrm{mmol}$ and their average affinity is $-6.36 \mathrm{kcal} / \mathrm{mmol}$. Additionally, 14 components and 19 targets of FZ have 266 docking results with the average affinity of -6.58 $\mathrm{kcal} / \mathrm{mmol}$, while 43 components and 27 targets of FZ have 1161 docking results with the average affinity of $-6.25 \mathrm{kcal} / \mathrm{mmol}$. PRKACA $(-7.30 \mathrm{kcal} / \mathrm{mmol})$, ADORA2A $(-7.20 \mathrm{kcal} / \mathrm{mmol})$ and GRIN2A $(-7.07 \mathrm{kcal} / \mathrm{mmol})$ are the top 3 genes with best average binding affinities, while the top 3 ligands with best average binding affinities are RS048 (-9.10 kcal/mmol), RS038 $(-9.00 \mathrm{kcal} / \mathrm{mmol})$ and FZ042 $(-8.61 \mathrm{kcal} / \mathrm{mmol})$. The top 3 binding relationship are PRKACA-RS048 (-11.30 $\mathrm{kcal} / \mathrm{mmol})$, AKT1- RS038 (-11.20 kcal/mmol) and AKT2- RS038 (-11.20 kcal/mmol). According to the visualization of ligands-receptors interactions (Fig. 10), there are hydrogen bonds, hydrophobic interactions and salt bridge between RS048 and PRKACA. And the interactions of RS038 and AKT1 includes hydrogen bonds, hydrophobic interactions, salt bridge and $\pi$-Cation interaction. Additionally, RS038 and AKT2 have the interactions of hydrogen bonds, hydrophobic interactions, salt bridge and $\pi$-Stacking (parallel). The above results indicate that both RS and FZ can effectively bind with the proteins contained in the comprehensive pathways, validating that RS and FZ play synergistic roles in the treatment of SSS.
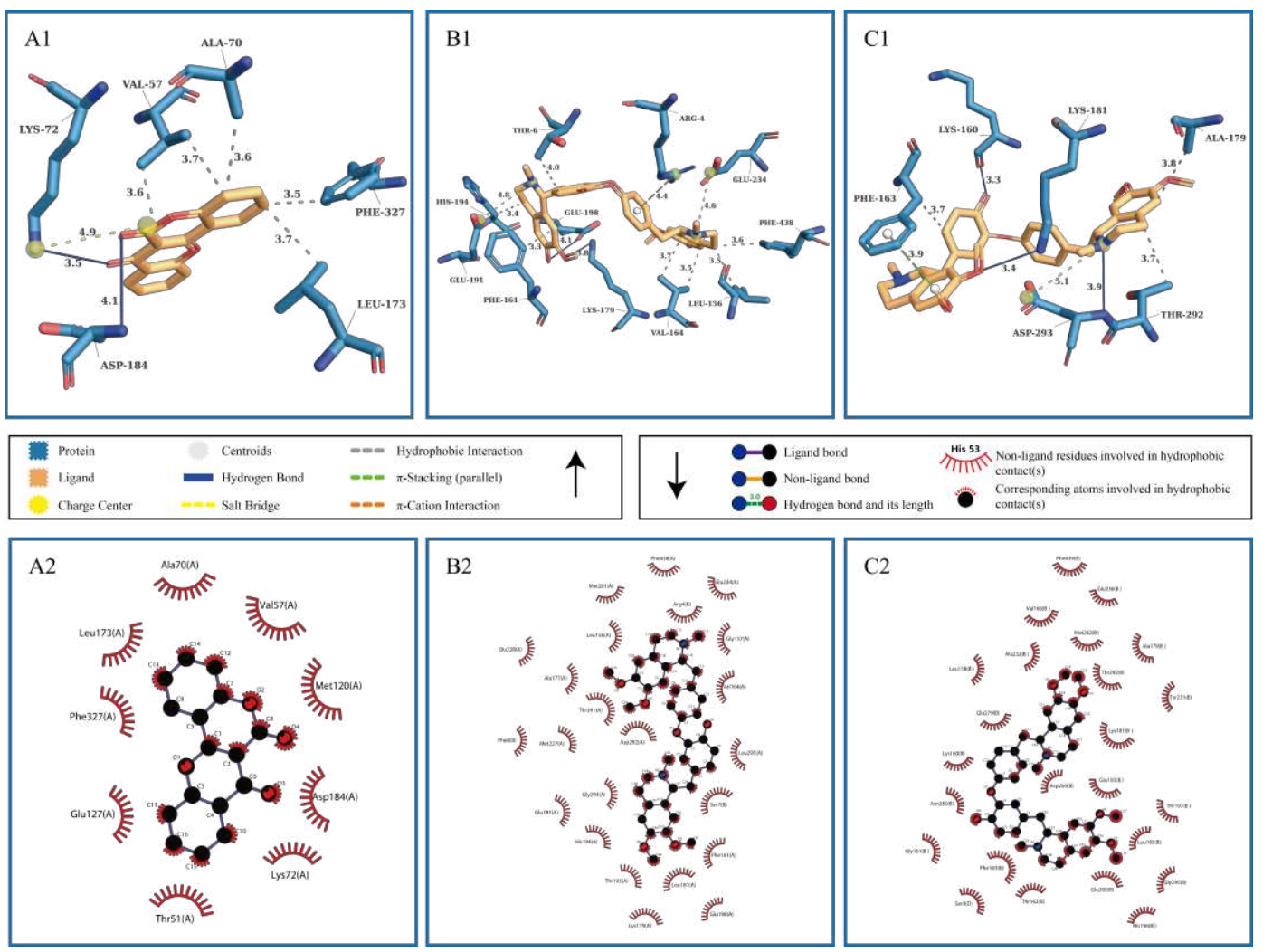

Fig. 10. The interactions visualization of receptors and ligands. (A1, A2) PRKACA-RS048, (B1, B2) AKT1-RS038, (C1, C2) AKT2-RS038.

\section{Discussion}

SSS is a common cardiovascular disease, which is related to many pathogenic factors, such as 
fibrosis of sinoatrial node and the surrounding tissues, ion channel dysfunction, sinoatrial node remodeling, etc. Related pathogenetic genes of SSS include SCN5A, HCN4, MYH6, etc. The related biological processes contain calcium signal, mitochondria and signal transduction, while the related pathways include neuroactive ligand-receptor interaction pathway, cAMP signaling pathway, calcium signaling pathway, PI3K-Akt signaling pathway, Rap1 signaling pathway, etc. Our research found that SFD can treat SSS by regulating biological processes and pathways mentioned above. We can primarily draw a conclusion of that the therapeutic mechanisms of SFD in treating SSS related to the regulation of signal transduction, ion channel functions, energy metabolism, etc. Therefore, the relationship between SSS and the above pathogenic factors was indirectly confirmed.

In this paper, we elaborate the synergistic mechanisms of SFD on SSS from four aspects. First of all, C-T network shows that RS and FZ have 419 identical targets, accounting for $40.68 \%$ of RS targets and $69.95 \%$ of FZ targets. This indicates that RS and FZ in SFD have potential synergistic therapeutic effect, which is the foundation of the following analysis. Secondly, SIC and TCO of each component in SFD were calculated, showing that SFCG consisted of 55 components are the key components of SFD and can contribute major effect of SFD in treating SSS, accounting for $90.05 \%$ of SIC and $90.02 \%$ of TCO. The results have proved that the effective therapeutic effect of SFD on SSS originates from all active components, not a few components. A further analysis showed that the targets interacting with SFCG could cover $90.86 \%$ enriched pathways of SFD targets and $92.50 \%$ of Reference KEGG, proving the reliability and accuracy of our proposed SFCG selecting model. Thirdly, GO and KEGG pathways enrichment analysis showed that there are 1051 shared enriched GO-BP terms and 117 enriched pathways between RS targets and FZ targets, which are closely related to SSS, proving that RS and FZ work synergistically in treating SSS, and meanwhile it indicates that RS and FZ also have their unique effect on SSS. Finally, pathway analysis proves once again that RS and FZ have synergistic effect on the treatment of SSS through the regulations of neuroactive ligand-receptor reaction, cAMP signaling pathway, calcium signaling pathway, PI3K-Akt signaling pathway and Rap1 signaling pathway. Molecular docking was used to validate the synergistic mechanisms of RS and FZ in treating SSS, showing that the components of SFCG were bound well to the targets involved in the comprehensive pathways with an average affinity of $-6.36 \mathrm{kcal} / \mathrm{mmol}$. All of these results indicate that RS and FZ in SFD can produce combined therapeutic effect on SSS.

\section{Conclusion}

In this study, we use system pharmacology, a novel effective pharmacological model and molecular docking to decode and validate the molecular mechanisms of SFD in treating SSS. The mechanism of SFD in treating SSS may be related to regulating ion channel function, signal transduction and energy metabolism, meanwhile, RS and FZ play a synergistic role in it. This strategy successfully integrated physicochemical properties of active components, network topological features, biological process analysis, pathway analysis and molecular docking analysis, thus provided novel ideas for TCM therapeutic mechanisms and prescription rules researches. 


\begin{abstract}
Abbreviations
SSS: Sick sinus syndrome; TCM: Traditional Chinese Medicine; SFD: Shenfu Decoction; SFCG: Synergistic functional components group; RS: Panax ginseng C.A.Mey; FZ: Aconiti Lateralis Preparata; SFI: Shenfu Injection; SIC: Synergistic influential contribution; TCO: Target coverage optimization; GI Absorption: Gastrointestinal Absorption; GO: Gene Ontology; KEGG: Kyoto Encyclopedia of Genes and Genomes; CC: Common components; BP: Biological processes.
\end{abstract}

\title{
Ethics Approval and Consent to Participate
}

Not applicable.

\section{Consent to Publish}

The manuscript is approved by all authors for publication.

\section{Availability of Data and Materials}

The data used or generated in this study are available from the corresponding author on reasonable request.

\section{Competing interests}

The authors declare that they have no known competing financial interests or personal relationships that could have appeared to influence the work reported in this paper.

\section{Funding}

This work was supported by the National Natural Science Foundation of China (No. 81774260), and Administration of Traditional Chinese Medicine of Guangdong Province of China (No. 20184011).

\section{Authors' Contributions}

Yihua Li, Rong Li and Daogang Guan provided the concept and designed the study. Yihua Li, Zhiyun Luo conducted the data analysis and wrote the manuscript. Yupeng Chen, Kexin Wang, Yi Li, Jieqi Cai provided technical supports. Xinjun Zhao, Qingmin Chu, Rong Li and Daogang Guan contributed to revising and proof-reading the manuscript. All authors read and approved the final 
manuscript.

\section{Acknowledgements}

Not applicable.

\section{Appendix A. Supplementary data}

Supplementary data (Table S1. The pathogenesis genes of SSS. Table S2. All components of SFD. Table S3. The whole C-T network of SFD. Table S4. The calculation results of SIC and TCO. Table S5. The molecular docking results.) related to this article can be found in the online version.

\section{References}

[1] Ewy, G.A. Sick sinus syndrome: synopsis. J. Am. Coll. Cardiol. 2014, 64, 539-540.

[2] Semelka, M.; Gera, J.; Usman, S. Sick sinus syndrome: a review. Am. Fam. Physician. 2013, 87, 691-696.

[3] Tian, R.; Zhang, Z.; Liu, K.; Hu, S.; Tang, Z.; Xie, C.; Lai, Z.; Liu, Z. Progress in the study of sick sinus syndrome. J. Gannan Med. Univ. 2019, 39, 662-667.

[4] Lai, Z.M.; Zhou, X.; You, T.G.; Wang, J.; Mai, S.Y. Treatment of 46 Cases of Sick Sinus Syndrome with Shenfu Decoction. J. Trad. Chin. Med. 2007, 8, 717-718.

[5] Fu, J.; Liu, H.C. Observation on therapeutic effect of Shenfu Decoction on 32 cases of senile sick sinus syndrome. Acta. Chin. Med. Pharm. 2004, 2, 33.

[6] Li, Y.H.; Yu, B.; Duan, Z.Z.; Akinyi, O.M.; Yu, J.H.; Zhou, K.; Zhang, Y.; Gao, X.M. The coronary dilation effect of shen fu injection was mediated through NO. PLOS ONE 2014, 9, e92415.

[7] Wu. J.; Wang, X.; Chung, Y.Y.; Koh, C.H.; Liu, Z.; Guo, H.; Yuan, Q.; Wang, C.; Su, S.; Wei, H. L-Type Calcium Channel Inhibition Contributes to the Proarrhythmic Effects of Aconitine in Human Cardiomyocytes. PLoS ONE 2017, 12, e0168435.

[8] Liu, C.; Hou, Y.; Wang, X.; Zhao, Z.; Liu, Z.; Zhai, J.; Mao, J.; Shang, H. Clinical assessment of Shenfu injection loading in the treatment of patients with exacerbation of chronic heart failure due to coronary heart disease: study protocol for a randomized controlled trial. Trials 2015, 16, 222.

[9] Liu, F. Clinical Effect of Shenfu Injection on Sick Sinus Syndrome. Chin. J. Clinical. Rational. Drug. Use. 2015, 8, 33-34. 
[10] Li, G; Liu, J. Shenfu Injection versus M Cholinoceptor Blockers for Sick Sinus Syndrome: A Systematic Review. Chin Pharm. 2014, 25, 4576-4579.

[11] Guo, W., Huang, J., Wang, N., Tan, H., Cheung, F., Chen, F., Feng, Y. Integrating Network Pharmacology and Pharmacological Evaluation for Deciphering the Action Mechanism of Herbal Formula Zuojin Pill in Suppressing Hepatocellular Carcinoma. Front. Pharmacol. 2019, 10,1185 .

[12] Li, S.; Zhang, B. Traditional Chinese medicine network pharmacology: theory, methodology and application. Chin. J. Nat. Med. 2013, 11, 110-120.

[13] Daina, A.; Zoete, V. A BOILED-Egg To Predict Gastrointestinal Absorption and Brain Penetration of Small Molecules. ChemMedChem. 2016, 11, 1117-1121.

[14] Vraníková, B.; Gajdziok, J. Bioavailability and factors influencing its rate. Ceska. Slov. Farm. 2015, 64, 7-13.

[15] Yu, G.; Wang, L.; Han, Y.; He, Q. clusterProfiler: an R package for comparing biological themes among gene clusters. Omics 2012, 16, 284-287.

[16] Shannon, P.; Markiel, A.; Ozier, O.; Baliga, N.S.; Wang, J.T.; Ramage, D.; Amin, N.; Schwikowski, B.; Ideker, T. Cytoscape: a software environment for integrated models of biomolecular interaction networks. Genome Res. 2003, 13, 2498-2504.

[17] O'Boyle, N.M.; Banck, M.; James, C.A.; Morley, C.; Vandermeersch, T.; Hutchison, G.R. Open Babel: An open chemical toolbox. J. Cheminform. 2011, 3, 33.

[18] Forli, S.; Huey, R.; Pique, M.E.; Sanner, M.F.; Goodsell, D.S.; Olson, A.J. Computational protein-ligand docking and virtual drug screening with the AutoDock suite. Nat Protoc 2016, $11,905-919$.

[19] Trott, O.; Olson, A.J. AutoDock Vina: improving the speed and accuracy of docking with a new scoring function, efficient optimization, and multithreading. J. Comput Chem. 2010, 31, 455461.

[20] Salentin, S.; Schreiber, S.; Haupt, V.J.; Adasme, M.F.; Schroeder, M. PLIP: fully automated protein-ligand interaction profiler. Nucleic Acids Res. 2015, 43, W443-447.

[21] Laskowski, R.A.; Swindells, M.B. LigPlot+: multiple ligand-protein interaction diagrams for drug discovery. J. Chem. Inf. Model. 2011, 51, 2778-2786.

[22] Butters, T.D.; Aslanidi, O.V.; Inada, S.; Boyett, M.R.; Hancox, J.C.; Lei, M.; Zhang, H. Mechanistic links between $\mathrm{Na}+$ channel (SCN5A) mutations and impaired cardiac pacemaking in sick sinus syndrome. Circ Res 2010, 107, 126-137.

[23] Mengesha, H.G.; Tafesse, T.B.; Bule, M.H. I(f) Channel as an Emerging Therapeutic Target for Cardiovascular Diseases: A Review of Current Evidence and Controversies. Front. Pharmacol. 2017, 8, 874. 
[24] Holm, H.; Gudbjartsson, D.F.; Sulem, P.; Masson, G.; Helgadottir, H.T.; Zanon, C.; Magnusson, O.T.; Helgason, A.; Saemundsdottir, J.; Gylfason, A.; Stefansdottir, H.; Gretarsdottir, S.; Matthiasson, S.E.; Thorgeirsson, G.M.; Jonasdottir, A.; Sigurdsson, A.; Stefansson, H.; Werge, T.; Rafnar, T.; Kiemeney, L.A.; Parvez, B.; Muhammad, R.; Roden, D.M.; Darbar, D.; Thorleifsson, G.; Walters, G.B.; Kong, A.; Thorsteinsdottir, U.; Arnar, D.O.; Stefansson, K. A rare variant in MYH6 is associated with high risk of sick sinus syndrome. Nat. Genet. 2011, $43,316-320$.

[25] Chen, S.N.; Lombardi, R.; Karmouch, J.; Tsai, J.Y.; Czernuszewicz, G.; Taylor, M.R.G.; Mestroni, L.; Coarfa, C.; Gurha, P.; Marian, A.J. DNA Damage Response/TP53 Pathway Is Activated and Contributes to the Pathogenesis of Dilated Cardiomyopathy Associated With LMNA (Lamin A/C) Mutations. Circ Res 2019, 124, 856-873.

[26] Torre-Amione, G.; Kapadia, S.; Lee, J.; Bies, R.D.; Lebovitz, R.; Mann, D.L. Expression and functional significance of tumor necrosis factor receptors in human myocardium. Circulation 1995, 92, 1487-1493.

[27] Zhang, J.; Wei, F.; Ding, L.; Wang, L.; Zhang, X.; Yu, L.; Liu, R.; Kuang, X.; Jiao, B.; Yang, B.; Fan, J. MicroRNA-1976 regulates degeneration of the sinoatrial node by targeting $\mathrm{Ca}(\mathrm{v}) 1.2$ and $\mathrm{Ca}(\mathrm{v}) 1.3$ ion channels. J. Mol. Cell. Cardiol. 2019, 134, 74-85.

[28] Mancuso, C.; Santangelo, R. Panax ginseng and Panax quinquefolius: From pharmacology to toxicology. Food Chem Toxicol. 2017, 107, 362-372.

[29] Irfan, M.; Kwak, Y.; Han, C.; Hyun, SH.; Rhee, M.H. Adaptogenic effects of Panax ginseng on modulation of cardiovascular functions. J. Ginseng Res. 2020, 44, 538-543.

[30] Kim, J.H.; Yi, Y.; Kim, M.; Cho, J.Y. Role of ginsenosides, the main active components of Panax ginseng, in inflammatory responses and diseases. J. Ginseng Res. 2017, 41, 435-443.

[31] Liu, Z.; Li, Z.; Liu, X. Effect of ginsenoside Re on cardiomyocyte apoptosis and expression of Bcl-2/Bax gene after ischemia and reperfusion in rats. J. Huazhong Univ. Sci. Technolog. Med. Sci. 2002, 22, 305-309.

[32] Wang, J.D.; Cui, Y.; Wang, J.G.; Li, H.; Dai, J.C.; Ma, H.Y. Protective effects of the ginseng saponin Rh3 pretreatment to caspase-3 of myocardial ischemia-reperfusion injury in rats. $J$. Changchun Univ. Tradit. Chin. Med. 2017, 33, 13-15.

[33] Leung, K.W.; Pon, Y.L.; Wong, R.N.S.; Wong, A.S.T. Ginsenoside-Rg1 induces vascular endothelial growth factor expression through the glucocorticoid receptor-related phosphatidylinositol 3-kinase/Akt and beta-catenin/T-cell factor-dependent pathway in human endothelial cells. J. Biol. Chem. 2006, 281, 36280-36288.

[34] Wu, J.; Sun, C.; Wang, R.; Li, J.; Zhou, M.; Yan, M.; Xue, X.; Wang, C. Cardioprotective effect of paeonol against epirubicin-induced heart injury via regulating miR-1 and PI3K/AKT pathway. Chem. Biol. Interact. 2018, 286, 17-25. 
[35] Wang, Y.; Geng, J.; Jiang, M.; Li, C.; Han, Y.; Jiang, J. The cardiac electrophysiology effects of higenamine in guinea pig heart. Biomed Pharmacother. 2019, 109, 2348-2356.

[36] Zhou, Y.P. Review and evaluation of water soluble active components of aconite acting on cardiovascular system. Pharmocol. Clin. Chin Mater. Med. 2011, 27, 106-110.

[37] Chá vez-Lara, B.; Ponce-López, M.T.; Bravo, G.; Pastelín, G. Pharmacologic study of dopamine catabolites on the contractility of isolated guinea pig myocardium. Arch Inst. Cardiol. Mex. 1989, 59, 367-373.

[38] Liu, X.X.; Jian, X.X.; Cai, X.F.; Chao, R.B.; Chen, Q.H.; Chen, D.L.; Wang, X.L.; Wang, F.P. Cardioactive $\mathrm{C}_{19}$-diterpenoid alkaloids from the lateral roots of Aconitum carmichaeli "Fu $\mathrm{Zi}$ ". Chem. Pharm. Bull (Tokyo).2012, 60, 144-149.

[39] Betrie, A.H.; Ayton, S.; Bush, A.I.; Angus, J.A.; Lei, P.; Wright, C.E. Evidence of a Cardiovascular Function for Microtubule-Associated Protein Tau. J. Alzheimers. Dis. 2017, 56, 849-860.

[40] Freyermuth, F.; Rau, F.; Kokunai, Y.; Linke, T.; Sellier, C.; Nakamori, M.; Kino, Y.; Arandel, L.; Jollet, A.; Thibault, C.; Philipps, M.; Vicaire, S.; Jost, B.; Udd, B.; Day, J.E.; Duboc, D.; Wahbi, K.; Matsumura, T.; Fujimura, H.; Mochizuki, H.; Deryckere, F.; Kimura, T.; Nukina, N.; Ishiura, S.; Lacroix, V.; Campan-Fournier, A.; Navratil, V.; Chautard, E.; Auboeuf, D.; Horie, M.; Imoto, K.; Lee, K.Y.; Swanson, M.S.; Munain, A.L.D.; Inada, S.; Itoh, H.; Nakazawa, K.; Ashihara, T.; Wang, E.; Zimmer, T.; Furling, D.; Takahashi, M.P.; CharletBerguerand, N. Splicing misregulation of SCN5A contributes to cardiac-conduction delay and heart arrhythmia in myotonic dystrophy. Nat. Commun. 2016, 7, 11067.

[41] Huston, J.M.; Tracey, K.J. The pulse of inflammation: heart rate variability, the cholinergic anti-inflammatory pathway and implications for therapy. J. Intern. Med. 2011, 269, 45-53.

[42] Lakatta, E.G., Maltsev, V.A., Vinogradova, T.M. A coupled SYSTEM of intracellular Ca2+ clocks and surface membrane voltage clocks controls the timekeeping mechanism of the heart's pacemaker. Circ Res 2010, 106, 659-673.

[43] Montaigne, D.; Maréchal, X.; Lacroix, D.; Staels, B. From cardiac mitochondrial dysfunction to clinical arrhythmias. Int. J. Cardiol. 2015, 184, 597-599.

[44] Wang, J.; Gareri, C.; Rockman, H.A. G-Protein-Coupled Receptors in Heart Disease. Circ Res 2018, 123, 716-735.

[45] Aziz, Q.; Finlay, M.; Montaigne, D.; Ojake, L.; Li, Y.; Anderson, N.; Ludwig, A.; Tinker, A. ATP-sensitive potassium channels in the sinoatrial node contribute to heart rate control and adaptation to hypoxia. J. Biol. Chem. 2018, 293, 8912-8921.

[46] DiFrancesco, D. The role of the funny current in pacemaker activity. Circ Res 2010, 106, 434446. 
[47] Xiao, B.; Tian, X.; Xie, W.; Jones, P.P.; Cai, S.; Wang, X.; Jiang, D.; Kong, H.; Zhang, L.; Chen, K.; Walsh, M.P.; Cheng, H.; Chen, S.R.W. Functional consequence of protein kinase Adependent phosphorylation of the cardiac ryanodine receptor: sensitization of store overloadinduced Ca2+ release. J. Biol. Chem. 2007, 282, 30256-30264.

[48] Braz, J.C.; Gregory, K.; Pathak, A.; Zhao, W.; Sahin, B.; Klevitsky, R.; Kimball, T.F.; Lorenz, J.N.; Nairn, A.C.; Liggett, S.B.; Bodi, L.; Wang, S.; Schwartz, A.; Lakatta, E.G.; DePaoliRoach, A.A.; Robbins, J.; Hewett, T.E.; Bibb, J.A.; Westfall, M.V.; Kranias, E.G.; Molkentin, J.D. PKC-alpha regulates cardiac contractility and propensity toward heart failure. Nat. Med. 2004, 10, 248-254.

[49] Abeyrathna, P.; Su, Y. The critical role of Akt in cardiovascular function. Vascul Pharmacol. 2015, 74, 38-48.

[50] Rose, B.A.; Force, T.; Wang, Y. Mitogen-activated protein kinase signaling in the heart: angels versus demons in a heart-breaking tale. Physiol. Rev. 2010, 90, 1507-1546.

[51] Bers, D.M. Cardiac excitation-contraction coupling. Nature 2002, 415, 198-205.

[52] Ghigo, A.; Laffargue, M.; Li, M.; Hirsch, E. PI3K and Calcium Signaling in Cardiovascular Disease. Circ Res 2017, 121, 282-292.

[53] Dong, W.; Yang, Z.; Yang, F.; Wang, J.; Zhuang, Y.; Xu, C.; Zhang, B.; Tian, X.; Liu, D. Suppression of Rap1 impairs cardiac myofibrils and conduction system in zebrafish. PLoS ONE 2012, 7, e50960.

[54] Vinogradova, T.M.; Lakatta, E.G. Regulation of basal and reserve cardiac pacemaker function by interactions of cAMP-mediated PKA-dependent $\mathrm{Ca} 2+$ cycling with surface membrane channels. J. Mol. Cell. Cardiol. 2009, 47, 456-474.

[55] Calkoen, E.E.; Vicente-Steijn, R.; Hahurij, N.D.; Munsteren, C.J.V.; Roest, A.A.W.; DeRuiter, M.C.; Steendijk, P.; Schalij, M.J.; Groot, A.C.G.; Blom, N.A.; Jongbloed, M.R.M. Abnormal sinoatrial node development resulting from disturbed vascular endothelial growth factor signaling. Int. J. Cardiol. 2015, 183, 249-257. 
Figures

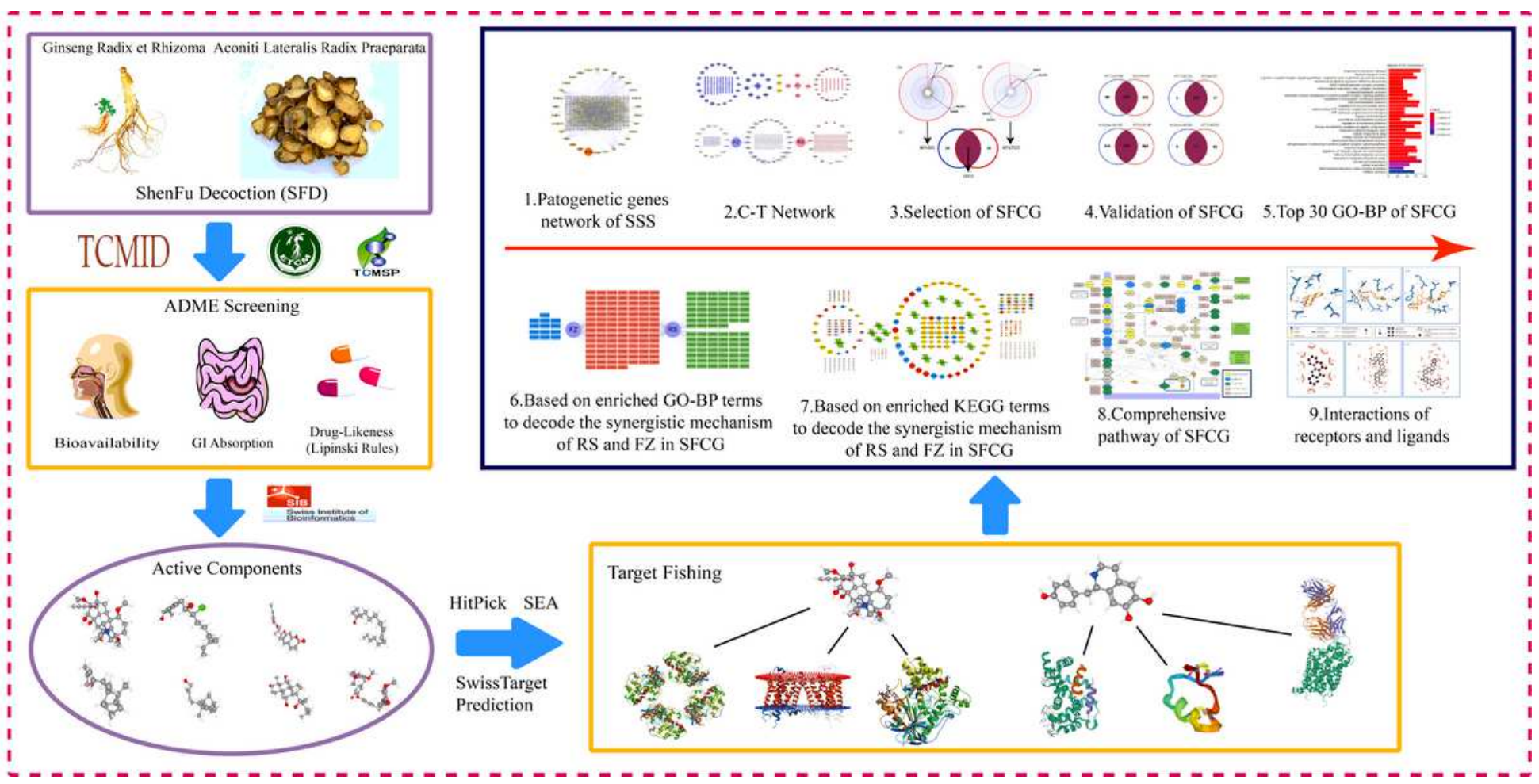

Figure 1

The flow chart of systematic pharmacology to decode the synergistic mechanism of SFD in treating SSS. 


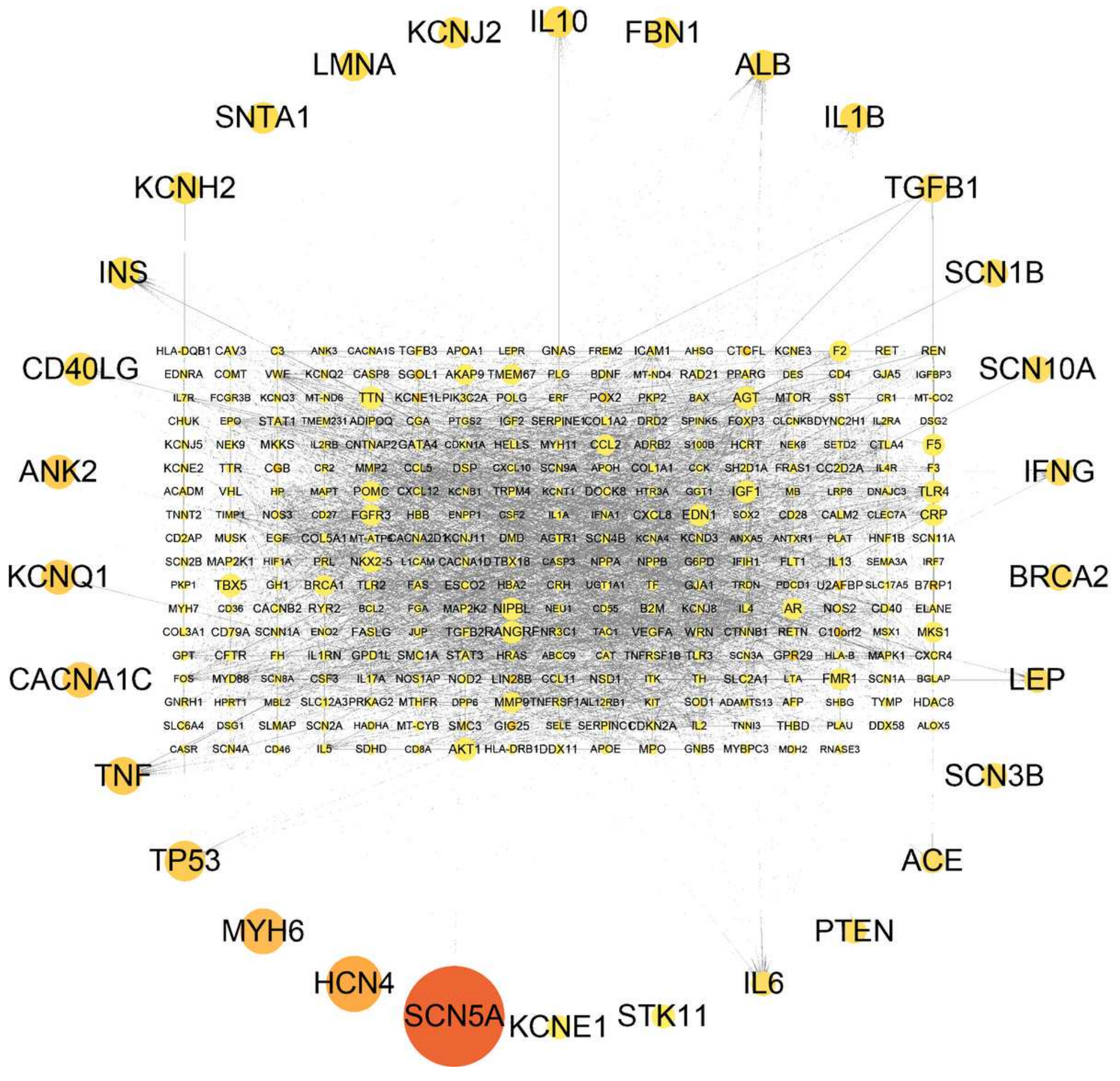

Figure 2

The weighted pathogenesis genes network of SSS. The node size means the weight of the node, which quantified by relevant score. 

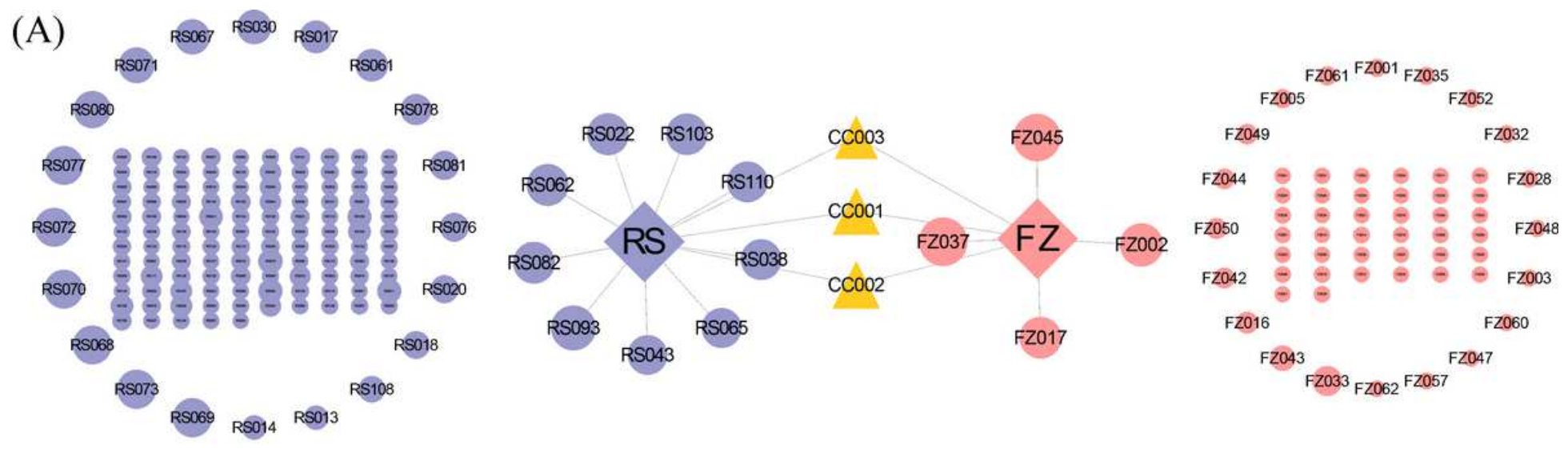

(B)
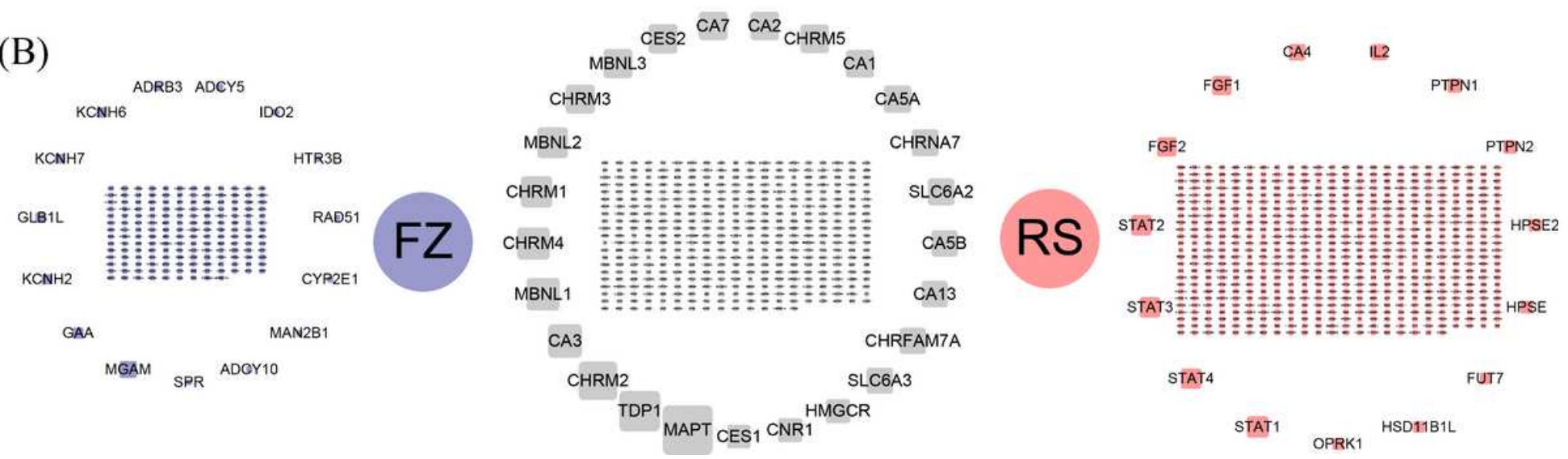

Figure 3

Components-Targets network of SFD. (A)Herbs-Components network. The node size means the number of genes that are targeted by the node. The blue nodes belong to RS, the pink nodes belong to FZ while the orange nodes are the common components of RS and FZ. (B)Herbs-Targets network. The node size represents the number of components target to the node. The blue nodes belong to $\mathrm{FZ}$, the pink nodes belong to RS while the grey nodes are the common targets of RS and FZ. 


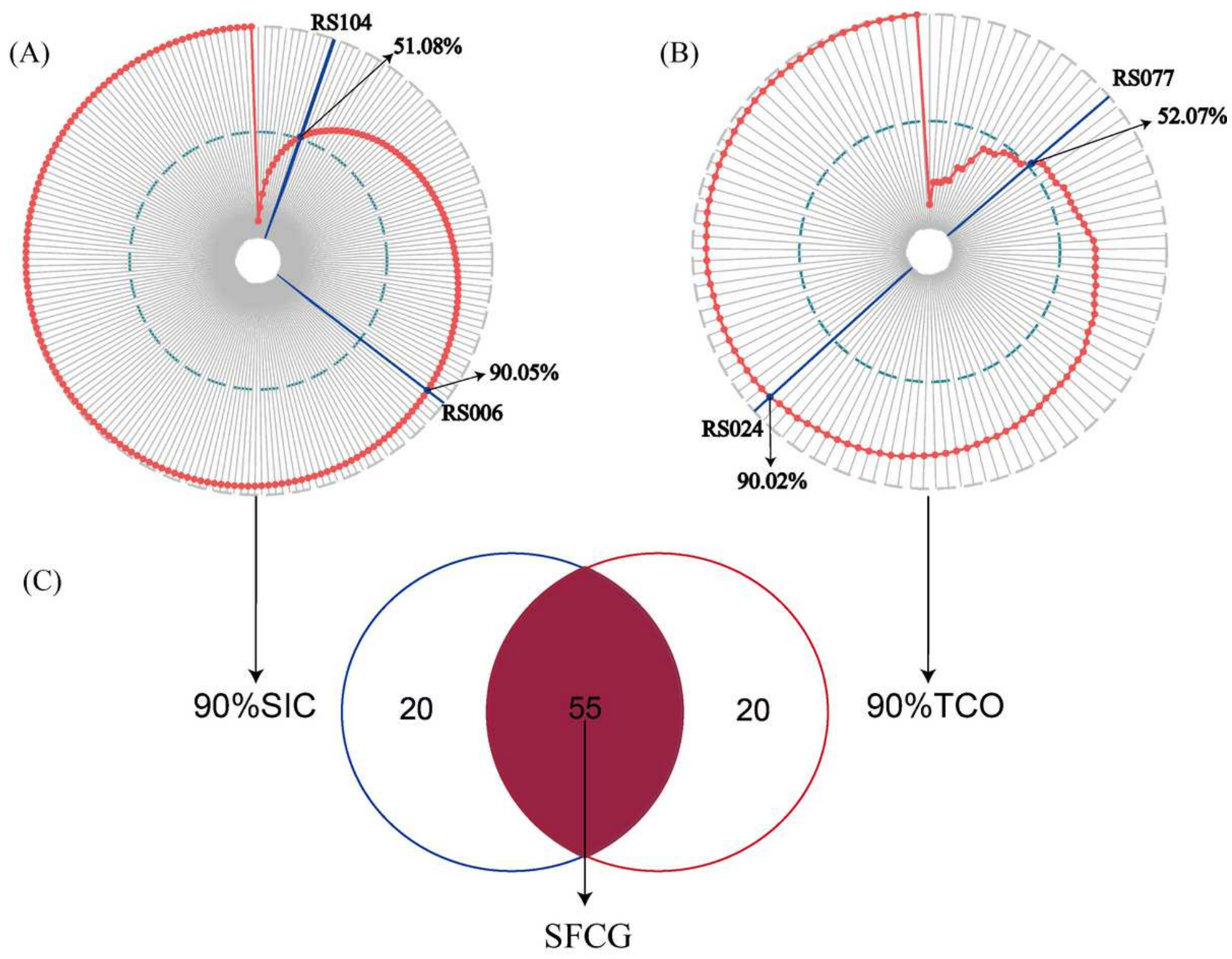

\section{Figure 4}

The calculation result of SIC and TCO. (A)The calculation result of SIC. (B) The calculation result of TCO. (C)The selected SFCG with 55 components. 

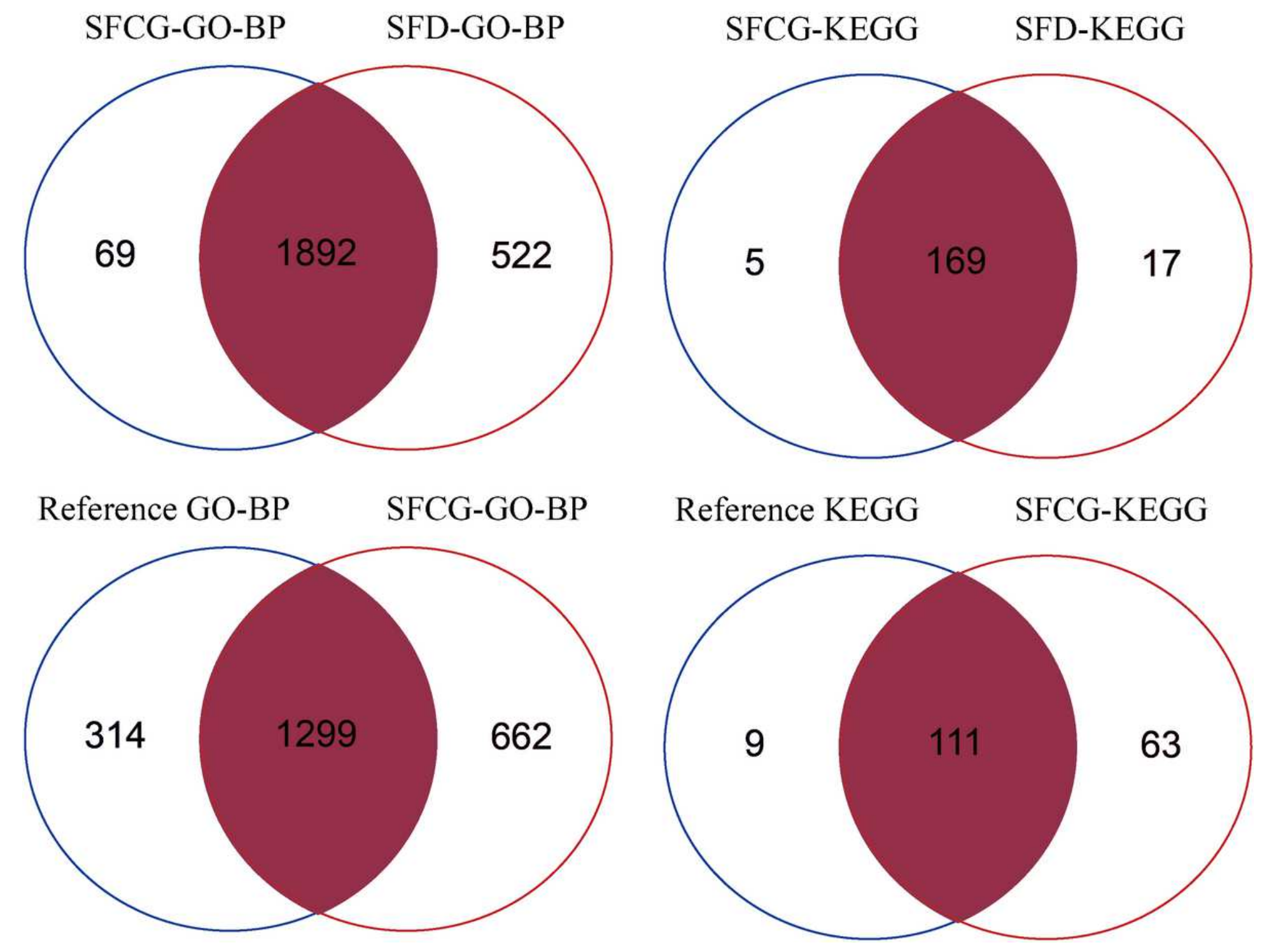

Figure 5

Functional validation of SFCG in treating SSS. 


\section{Barplot of GO Enrichment}

electron transport chain

G protein-coupled receptor signaling pathway, coupled to cyclic nucleotide second messenger mitochondrial electron transport, NADH to ubiquinone $\mathrm{NADH}$ dehydrogenase complex assembly mitochondrial respiratory chain complex I assembly icosanoid metabolic process adenylate cyclase-modulating $\mathrm{G}$ protein-coupled receptor signaling pathway regulation of postsynaptic membrane potential fatty acid metabolic process regulation of neurotransmitter levels mitochondrial ATP synthesis coupled electron transport ATP synthesis coupled electron transport organic anion transport arachidonic acid metabolic process regulation of membrane potential energy derivation by oxidation of organic compounds respiratory electron transport chain cellular response to drug cellular calcium ion homeostasis unsaturated fatty acid metabolic process phospholipase $\mathrm{C}$-activating $\mathrm{G}$ protein-coupled receptor signaling pathway response to lipopolysaccharide regulation of cytosolic calcium ion concentration fatty acid derivative metabolic process response to molecule of bacterial origin calcium ion homeostasis cellular respiration mitochondrial respiratory chain complex assembly rhythmic process

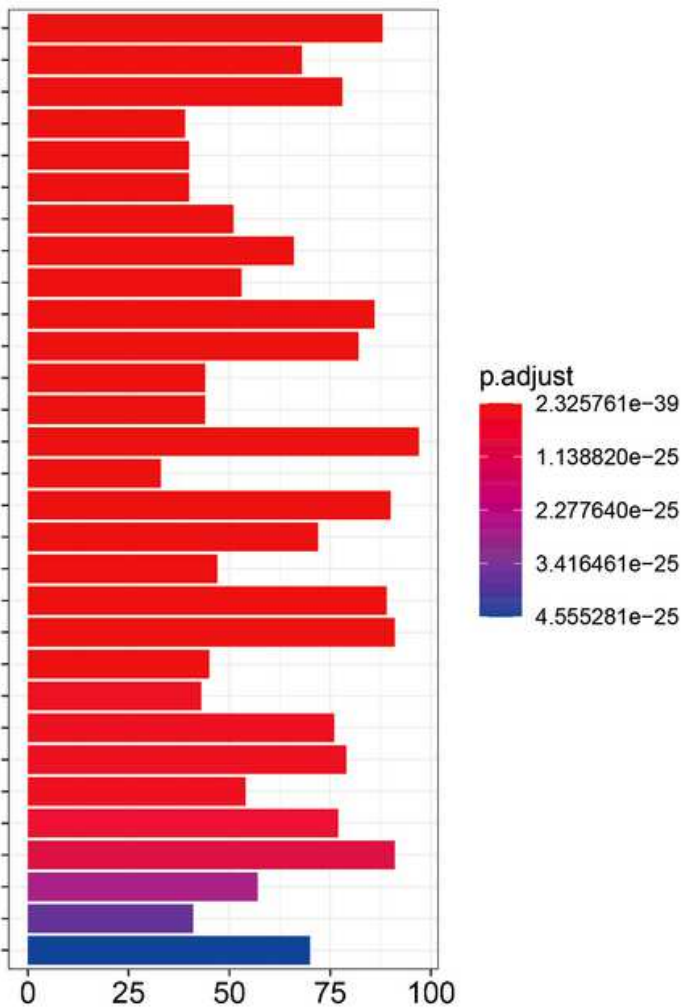

\section{Figure 6}

The top 30 enriched GO-BP terms of SFCG targets.

\begin{tabular}{|c|c|c|c|c|c|c|c|c|c|c|c|c|c|c|c|}
\hline & 60.0046890 & 60.0032490 & 60.0035296 & 60.00003012 & 60.0072593 & 60.0007568 & 60.0033002 & 60.0071902 & 60.0019229 & 60.0048683 & 60.0060249 & 60.0032147 & 60:1904658 \\
\hline & & & 60.0099177 & 60.2000377 & 60.0010518 & $60: 0003014$ & Go: 1003532 & 60.0062012 & $60: 0048511$ & 60.0010742 & 60.0032409 & 60.0050900 & 60.1903034 & 60.0062013 & 360.0106100 \\
\hline & & & 60.0097755 & 60.0050708 & 60.0006979 & | $60: 00043434$ & 60.0055074 & 60.0009914 & 60:0002237 & 60.00033312 & 600072511 & | 60.0050999 & 60.0015749 & 60:0051924 & 4 60.0030168 \\
\hline & & & 60.0048659 & 60.0050080 & 60.0008217 & 60:0019233 & 60.000110 & 60:0019216 & 60.00510000 & 60.0002283 & 60.0061049 & 1. 60.1904062 & 60.0007596 & 60.0001508 & 60.0006816 \\
\hline & 60.0030198 & & 60.0097746 & 80.0097305 & 60.0050006 & [ 60:2000379 & 60.0034765 & 60.0001659 & 60.0031044 & 60.0010959 & 60.0032768 & 60.0043405 & $60: 1903409$ & 60.0007584 & 60.0071216 \\
\hline 60.0043271 & | 60.0000037 & 60.0048638 & 60.0060191 & 60.0010039 & 60.0045834 & 60.00035094 & 60.0009314 & 600072503 & 60.0009612 & 60.0060401 & 60.0031098 & 60.0008808 & 60.009007 & 60.0002446 & 60.0043406 \\
\hline 0.0050795 & 00.0009205 & 0.00902 & $60: 0051222$ & 60.0048677 & 60.0019932 & 60.0010870 & $60: 1004951$ & 60.0032355 & 60:0006874 & 60.0071219 & 60.0042119 & 60.0070374 & 60.0048732 & & \\
\hline 0.045444 & 600043112 & 0.0035264 & -60.0098657 & -60.0031007 & -60.0007813 & 60.0000193 & 00.0045770 & 60.0006879 & 60.0043270 & & & & & & \\
\hline & 00430 & & G0:0010517 & $60: 0014074$ & $60: 0042139$ & |co:0032868 & 60.0048860 & | 60.0050804 & | Go:1901853 & 60.0042593 & 60.0034599 & G0.0008565 & 60:0070372 & $60: 0018212$ & 60.0060402 \\
\hline & & & $60: 0070997$ & 60.0018105 & $60: 0042310$ & | 60.0008202$\}$ & G0:1903522 & | $60: 0003073$ & 60.0006868 & 60.1990845 & 60.0049651 & 60:0032412 & 60.051403 & $60: 0000326$ & 60.0022898 \\
\hline & & & G0:1901654 & 60:0007611 & 60:0104004 & | 60.0006039 & G0.0007831 & |c0:0071214 & 600007204 & 60.0033273 & 602001057 & 60.0019722 & 60:0097237 & 60:1901216 & 60.0070838 \\
\hline & & & $60: 0070482$ & 60.0046833 & $60: 0045471$ & | $60: 00032293$ & 60.0051047 & 60:000232391 & 60.0035150 & 60.0000276 & 60.0034219 & 60.0050728 & 60.0051048 & $60: 0007599$ & 60.0050817 \\
\hline & & & $60: 0042138$ & 60.0051341 & $60: 0050727$ & | 60:0150076 & 60:0001666 & 60:0046545 & 60.0002791 & 60.0071496 & 60.0050796 & 60.0046209 & 60.0000187 & 60:0120161 & 60.0032102 \\
\hline & & & GO-0044706 & 60.0035890 & 60.0062197 & | 60.00001505 & Go:0048371 & 60:0006936 & 60.0097756 & 60.0071222 & 60.0009743 & 60.0071375 & 60.0070588 & 60.0045862 & 2. 60.1901214 \\
\hline & & & 60.0051480 & $60: 1903524$ & G0:0003018 & 60.0000302 & & & & $60: 0018108$ & 60.0030193 & 60:0050886 & 60.0033500 & $60: 0051235$ & 60:0070371 \\
\hline
\end{tabular}

\section{Figure 7}

The upper quartile of GO-BP terms of RS and FZ. The blue and green rectangular nodes are the unique GO-BP terms of FZ targets and RS targets respectively, while the red ones are the shared GO-BP terms of RS targets and FZ targets. 


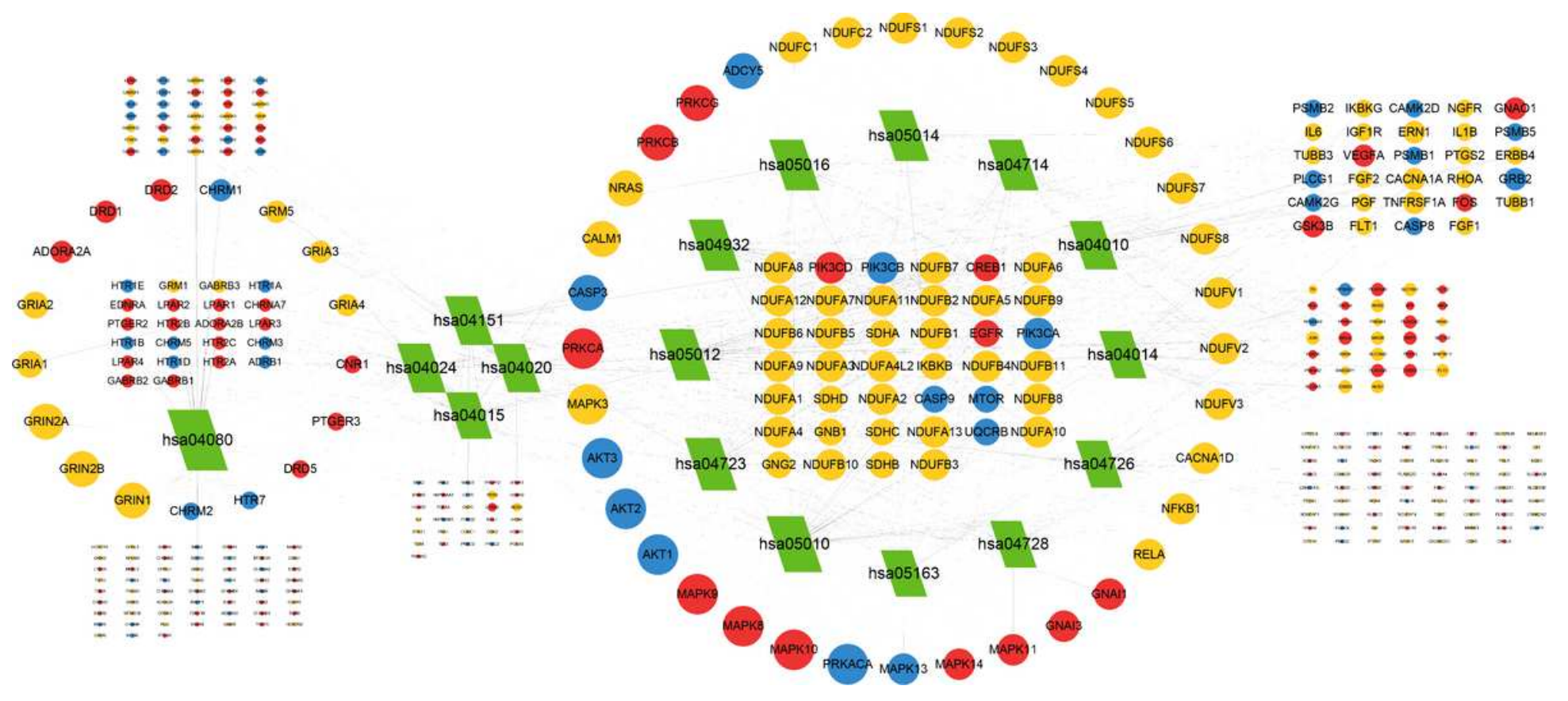

\section{Figure 8}

Targets-Pathways network of SFD. The orange and blue nodes are the unique targets of RS and FZ respectively, while the red ones are the shared targets of RS and FZ. The green nodes are the pathways of targets of SFD. 


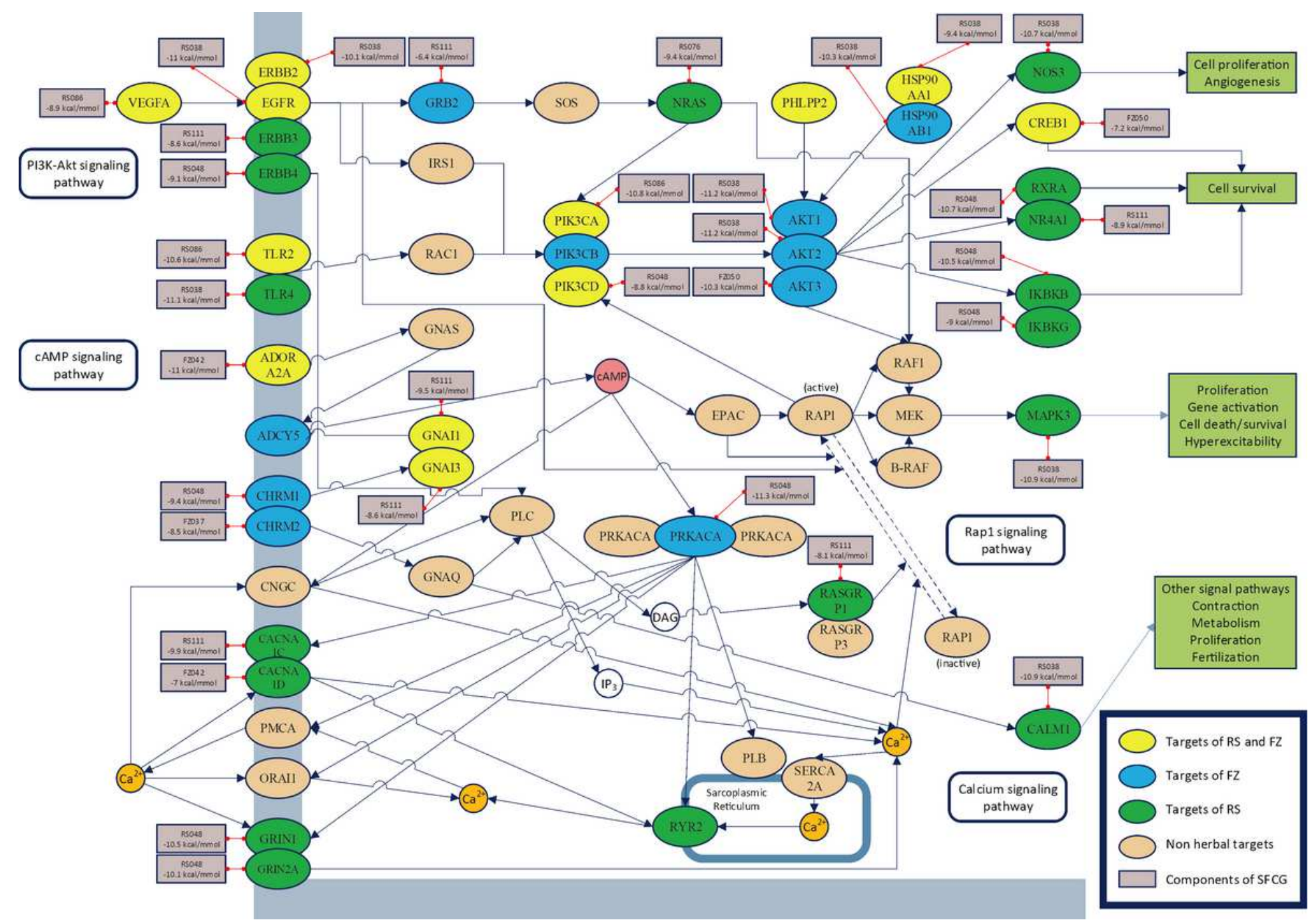

Figure 9

The comprehensive pathway of SFD in treating SSS. 

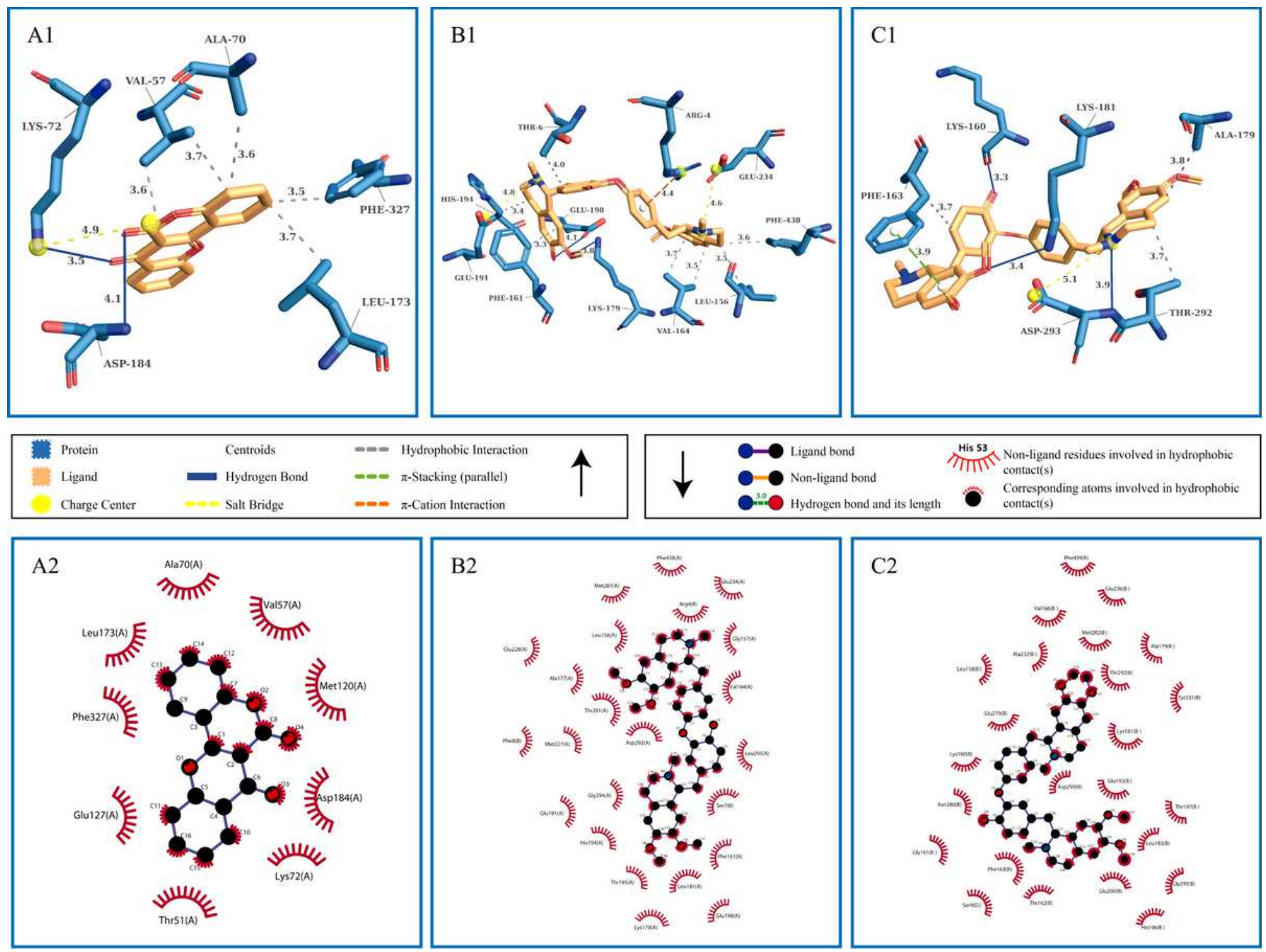

Figure 10

The interactions visualization of receptors and ligands. (A1, A2) PRKACA-RS048, (B1, B2) AKT1-RS038, (C1, C2) AKT2-RS038.

\section{Supplementary Files}

This is a list of supplementary files associated with this preprint. Click to download.

- TableS1ThepathogenesisgenesofSSS.docx

- TableS2AllcomponentsofSFD.docx

- TableS3ThewholeCTnetworkofSFD.xlsx

- TableS4ThecalculationresultsofSICandTCO.docx

- TableS5Themoleculardockingresults.xIsx 